\title{
Structural, magnetostatic, and magnetodynamic studies of Co/Mo-based uncompensated synthetic antiferromagnets
}

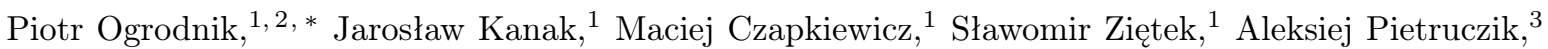 \\ Krzysztof Morawiec, ${ }^{3}$ Piotr Dłużewski, ${ }^{3}$ Krzysztof Dybko, ${ }^{3}$ Andrzej Wawro, ${ }^{3}$ and Tomasz Stobiecki ${ }^{1,4}$ \\ ${ }^{1}$ AGH University of Science and Technology, Department of Electronics, al. A. Mickiewicza 30, 30-059 Krakow, Poland \\ ${ }^{2}$ Warsaw University of Technology, Faculty of Physics, ul. Koszykowa 75, 00-662 Warsaw, Poland \\ ${ }^{3}$ Institute of Physics, Polish Academy of Sciences, \\ Aleja Lotników 32/46, PL-02668 Warsaw, Poland \\ ${ }^{4}$ AGH University of Science and Technology, Faculty of Physics and \\ Applied Computer Science, Aleja Mickiewicza 30, 30-059 Krakow, Poland
}

(Dated: October 18, 2019)

\begin{abstract}
In this work, we comprehensively investigate and discuss the structural, magnetostatic, dynamic, and magnetoresistive properties of epitaxial $\mathrm{Co} / \mathrm{Mo}$ superlattices. The magnetization of the Co sublayers is coupled antiferromagnetically with a strength that depends on the thickness of the nonmagnetic Mo spacer. The magnetization and magnetoresistance hysteresis loops clearly reflect interlayer exchange coupling and the occurrence of uniaxial magnetic anisotropy induced by the strained Co sublayers. Upon accounting for a deviation of the sublayer thicknesses from the nominal value, theoretical modeling, including both micromagnetic and macrospin approaches, precisely reproduces experimental magnetic hysteresis loops, magnetoresistance curves, and ferromagnetic resonance dispersion relations. The Mo spacer thickness as a function of the interlayer magnetic coupling is determined as a fitting parameter by modeling the experimental results.
\end{abstract}

PACS numbers: 75.47.-m, 76.50.+g, 75.78.-n, 75.75.-c

\section{INTRODUCTION}

Magnetic superlattices arising from the abundant contribution of atoms forming interfaces and from the nonmagnetic layers being thinner than the charge- and spintransport characteristic lengths offer enhanced or completely new properties in comparison with the bulk materials. In particular, such multilayers have attracted a great deal of attention after the discovery of interlayer exchange coupling [1], which was observed in systems magnetized both in the layer plane [4 or perpendicularly to the layer plane [5]. The interlayer coupling was explained within the framework of RudermanKittel-Kasuya-Yosida (RKKY) theory [6, with quantum interference resulting from spin-dependent reflections of Bloch waves at the paramagnet-ferromagnet interfaces being due to confinement by ultrathin layers [7] or dipolar interactions. Due to this type of coupling, the alignment of magnetization was usually constrained to collinear or perpendicular configurations in the magnetic sublayers. The discovery of interlayer exchanged-coupled systems was followed by that of a markedly enhanced spin-dependent transport effect called "giant magnetoresistance" (GMR) [8, 9].

A strong antiferromagnetic coupling between two ferromagnetic layers separated by a thin nonmagnetic spacer (e.g., Ru) enabled the fabrication of synthetic antiferromagnets (SAFs) 10 12. Recently, multilayer SAF systems combined with a metal layer exhibiting strong spin-

\footnotetext{
*Electronic address: piotr.ogrodnik@pw.edu.pl
}

orbit interactions such as $\mathrm{Pt} / \mathrm{Fe}_{20} \mathrm{Ni}_{80} / \mathrm{Ru} / \mathrm{Fe}_{20} \mathrm{Ni}_{80}[13$ or $\mathrm{Pt} /(\mathrm{Co} / \mathrm{Pd}) / \mathrm{Ru} /(\mathrm{Co} / \mathrm{Pd})$ 14 were used to demonstrate, particularly in patterned devices, an electrically detected spin-orbit torque ferromagnetic resonance (SOT-FMR) and magnetization switching between highand low-resistance configurations. In addition, $\mathrm{Wu}$ et al. [15] recently demonstrated SOT-induced fieldfree magnetization switching in an exchange-coupled i$\mathrm{CoFeB} / \mathrm{Mo}$ (wedged) $/ \mathrm{p}-\mathrm{CoFeB}$ structure, where the letter "i" ("p") stands for in-plane (perpendicular) magnetic anisotropy.

The magnetostatic properties of superlattices, with regular or more sophisticated structures, can be also analyzed by dynamic techniques, such as by ferromagnetic resonance (FMR) [16-21. Such research seems to be particularly important provided the increase and tunability of FMR frequencies permit the design of novel ultrafast magneto-electronic devices [22]. Of the many types of superlattices, the heavy-metal/ferromagnetic $(\mathrm{HM} / \mathrm{FM})$ systems are investigated in particular because they exhibit interfacial Dzyaloshinsky-Moriya interactions (DMIs) and DMI-related phenomena (e.g., the formation of magnetic skyrmions) [23, 24]. Although the $\mathrm{Co} /$ Mo system belongs to the HM/FM group, it is still rather poorly recognized.

Current studies mainly focus on how structural evolution correlates with magnetic properties [25[30]. In addition, coupling effects have been reported in several papers. For example, Parkin showed that oscillatory exchange coupling was a typical feature for numerous transition-metal spacers [31]. The coupling strength increases both with the electron occupation of the $d$ shell and along each column in the periodic table. In 
addition, well-defined coupling with induced in-plane anisotropy was observed in sputter-deposited multilayers [32, and the structural and electronic factors affecting the coupling were analyzed theoretically for transitionmetal spacers, including Mo 33. However, this research did not quantitatively address the interlayer coupling strength.

In previous work we thoroughly investigated the magnetic properties (magnetic ordering and reversal processes) of $\mathrm{Co} / \mathrm{Mo} / \mathrm{Co}$ trilayers, both deposited on and covered by $\mathrm{Mo}$ or $\mathrm{Au}$ layers. The $\mathrm{Au}$ buffer invokes isotropic behavior of the $\mathrm{Co} / \mathrm{Mo} / \mathrm{Co}$ trilayers. For a thinner Co layer, the system displays perpendicular magnetization with oscillations between ferromagnetic (FM) and antiferromagnetic (AFM) alignments with $d_{\mathrm{Mo}}$, with the coupling field reaching as high as $3 \mathrm{kOe}$ [34. For thicker $d_{\mathrm{Co}}$, canted magnetization tuned by the coupling strength is observed. Conversely, due to a different crystalline symmetry and a lattice mismatch at the interfaces, the Mo buffer induces anisotropic strains that result in in-plane twofold magnetic anisotropy [35]. Moreover, the in-plane magnetized trilayers exhibit an AFM interlayer coupling in the Mo spacer thickness $\left(d_{\mathrm{Mo}}\right)$ ranging from 0.5 to $1.0 \mathrm{~nm}$. This coupling can be tuned or switched to FM type by ion irradiation [36, 37. Such an intended varying of the interlayer coupling enables periodic modification of the magnetization on the nanometer scale, which, in turn, allows Co/Mo multilayer systems to be fabricated as switchable magnonic crystals 37. More-detailed synchrotron research on Co/Mo multilayered systems revealed a possible contribution of induced magnetic moments at Mo atoms of the spacer to the observed coupling, as reported recently [38]. Furthermore, it has been shown that Fe dopants in the Mo spacer suppress the interlayer coupling strength [39].

In the present work we investigate the structural, magnetic static and dynamic, as well as field-dependent transport properties of $\mathrm{Co} /$ Mo epitaxially grown superlattices with a greater number of bilayers. The magnetization of the Co sublayers couples antiferromagnetically, forming the SAF structure. To date, Parkin [31] has produced the only publication available that discusses interlayer exchange coupling in $\mathrm{Co} / \mathrm{Mo}$ multilayers and considers the superlattice with 16 bilayers. In his work, $\mathrm{Co} / \mathrm{Mo}$ bilayers were sputter deposited, and no detailed structural or dynamical studies are available for such systems. We show herein that the symmetry and lattice-parameter mismatches at the interfaces generate anisotropic strain in the Co layers. As a consequence, magnetic anisotropy is induced in the sample plane with two mutually orthogonal axes of magnetization (easy and hard). Thus, the superlattices are characterized by both tunable interlayer coupling and in-plane anisotropy, which strongly defines the magnetization orientation. In addition, we consider the layer thickness and distribution of magnetic parameters, which is an immanent feature of real multilayers and is usually not accounted for in interpretations of acquired results. This experimental study is supported by micromagnetic and macrospin numerical simulations of magnetostatic and magnetodynamic SAF properties.

The paper is organized as follows: Section II provides details of sample fabrication, and Sec. III explains the experimental techniques used to characterize the samples. Section IV analyzes the structural data from high-angle $\mathrm{x}$-ray diffraction (XRD) and low-angle x-ray reflectometry (XRR) measurements. Section V describes the magnetic properties of $\mathrm{Co} /$ Mo superlattices and is divided into several subsections: It starts with a presentation of the theoretical macrospin model in Sec. VA1. The macrospin model has been validated by OOMMF micromagnetic calculations [40], which are briefly described in Sec. VA2, The results obtained from vibrating sample magnetometry (VSM) and four-probe magnetoresistance (MR) measurements are presented in Sec. V B 1. Section $\mathrm{VC}$ discusses in detail the magnetic dynamics in terms of FMR resonant modes, while the related results on interlayer coupling are given in Sec. VD Finally, Sec VI concludes and summarizes the paper.

\section{SAMPLE PREPARATION}

Epitaxial Co/Mo superlattices were deposited onto (11-20)-oriented sapphire wafer substrates by using a molecular-beam epitaxy system (Prevac). All samples contained five $\mathrm{Co} / \mathrm{Mo}$ bilayers in the following structures: $\quad S / \mathrm{V}(2.5) / \mathrm{Mo}(0.6) /[\mathrm{Co}(2.1) / \mathrm{Mo}(0.6)] \times$ $4 / \mathrm{Co}(2.1) / \mathrm{Mo}(0.6) / \mathrm{V}(3.0)$ (called the "C1 coupled sample"), $\quad S / \mathrm{V}(2.5) / \mathrm{Mo}(0.6) /[\mathrm{Co}(3.5) / \mathrm{Mo}(0.7)] \times$ $4 / \mathrm{Co}(3.5) / \mathrm{Mo}(0.6) / \mathrm{V}(3.0)$ (called the "C2 sample"), and $S / \mathrm{V}(2.5) / \mathrm{Mo}(0.6) /[\mathrm{Co}(1.5) / \mathrm{Mo}(0.9)] \times$ $4 / \mathrm{Co}(1.5) / \mathrm{Mo}(0.6) / \mathrm{V}(3.0)$ [called the "weakly coupled (WC) sample"]. The nominal thicknesses given above are in nanometers, and $S$ denotes a sapphire substrate $\left(\mathrm{Al}_{2} \mathrm{O}_{3}\right)$. The seed layer was thin $\mathrm{V}(110)$ because its crystalline structure is compatible with the substrate. The outer Co layers were also surrounded by thin Mo layers on the bottom and top to maintain the same type of interfaces. The entire stack was then covered again by a V capping layer. Except for the V buffer (deposited at room temperature and then annealed at $500^{\circ} \mathrm{C}$ for 3 $\mathrm{h}$ ), the remaining parts of the multilayered structures were deposited at room temperature. The component materials were evaporated from electron guns at rates of $0.05 \mathrm{~nm} / \mathrm{s}$, as measured by a quartz balance and a Hiden cross beam source. The crystalline structure was monitored in situ by reflection high-energy electron diffraction.

\section{EXPERIMENT}

The sample structure was characterized by using a high-resolution X'Pert-MPD diffractometer with a $\mathrm{Cu}$ anode. The samples were analyzed by using XRR and XRD. The diffractometer was equipped with an Euler 
cradle stage that allowed the sample to be rotated around the axis perpendicular to its surface and to be tilted from horizontal to vertical. This configuration allowed us to measure $\phi$ scans (sample rotation) at fixed $2 \theta$ and $\psi$ (sample tilt) to determine the orientation of the crystallites with respect to the sample plane. Additionally, the Euler cradle allowed us to make grazing-incidence x-ray diffraction measurements, which provided information on interplanar distances in the direction parallel to the sample surface.

The high-angle annular dark-field (HAADF) scanning transmission electron microscopy study was done by using a Titan Cubed 300 microscope operating at $300 \mathrm{kV}$ and equipped with an energy-dispersive x-ray (EDX) spectrometer. The cross-sectional lamellas were fabricated by using a focused Ga ion beam in a HeliosNanoLab system. Prior to preparing the lamellas, a Pt layer was deposited by using an electron gun to protect the sample surface from sputtering by Ga ions.

The magnetization hysteresis loops were measured in a high and low magnetic field by using VSM to determine a saturation magnetization and characteristic loops typical of coupled magnetic layers.

The room-temperature MR measurements were done on unpatterned, as-grown samples. Standard four-probe in-line contact alignment was implemented by using spring-type pins connected directly to the sample surface. This configuration is commonly called "current in plane." The magnetic field applied in the sample plane was rotated with respect to the direction of the current.

The FMR spectra were acquired by using a HP $8720 \mathrm{C}$ vector network analyzer (VNA). Samples were placed face down on a $50-\Omega$-matched coplanar waveguide with a line width of $500 \mu \mathrm{m}$. Microwave transmission was measured at a constant frequency (ranging from 5 to 16 $\mathrm{GHz}$ ) by sweeping the in-plane external magnetic field. The analysis of the relatively rich FMR spectra allowed us to determine the dispersion relations $f\left(H_{\text {res }}\right)$, which are broadly discussed in Sec. $\mathrm{VC}$

\section{STRUCTURAL STUDIES}

This section first shows that the considered $\mathrm{Co} / \mathrm{Mo}$ SAF forms a high-quality superlattice with welldistinguished sublayers with sufficiently sharp interfaces. Next, the thicknesses of all sublayers are determined with the help of XRR and XRD measurements and relevant simulations. Last but not least, the origin of the in-plane uniaxial magnetic anisotropy in Co layers is discussed and related to the distortion of hexagonal Co cells caused by the strain at the $\mathrm{Co} / \mathrm{Mo}$ interfaces.

Figure 1(a) shows a low-magnification TEM image of the C2 multilayer. AFM interlayer magnetic coupling occurs in this sample and is discussed later in this work. The layered Co/Mo structure is composed of five Co layers, separated distinctly by the Mo spacers. The Mo layers are visible in the brightest color. The whole lay- ered stack is deposited on the flat surface of the $\mathrm{V}$ buffer [denoted $\mathrm{V}(\mathrm{b})]$ and also capped by a $\mathrm{V}$ film $[\mathrm{V}(\mathrm{c})]$. The grainy structure in the upper part of the image illustrates the $\mathrm{Pt}$ covering layer that was deposited to prepare a sample slice for observation by transmission electron microscopy (TEM). The Mo layers seem to be continuous and relatively smooth. The columnar growth of Co and Mo layers results in greater roughness for the higher Mo layers. The presence of continuous Mo spacers is confirmed by a negligible influence of pinholes on AFM coupling, as discussed later in this work.

A closer look into the internal structure of the $\mathrm{Co} / \mathrm{Mo}$ multilayer reveals a parallel alignment of atomic layers expanding across the layered structure of the sample [Fig. 1(b)]. According to data from reflection high-energy electron diffraction (data not shown) and XRD (discussed later in this work), this observation confirms the epitaxial growth of the sample. The atomic planes parallel to the sample surface consist of bcc V (110), bcc Mo (110), and closed-packed hcp Co. The parallel alignment of the atomic layers across the entire sample is confirmed by the distinct spots in the Fourier transform shown in the inset of Fig. 1(c), which presents the details of the multilayered sample structure. The in-depth chemical profile obtained from EDX spectroscopy is correlated with the TEM image. The maxima in the $\mathrm{V}$ profile correspond to the cap layer (left) and the buffer (right), and the intensity oscillations in the Co and Mo signal are very distinct. The lower-intensity signals are from the very thin (four atomic layers) Mo spacer. Electrons from the e-beam centered at the Mo spacer are scattered in the sample and excite Co atoms in the vicinity of the interfaces. Details of the in-depth profiles are provided by high-angle annular dark-field measurements (black line). The fine structure reflects atomic layers of the component films. Finally, the broad peaks correspond to the Mo spacers.

The layered structure of the samples were investigated by using the XRR technique. Figure 2 shows reflectivity curves and associated numerical fits for the $\mathrm{C} 1, \mathrm{C} 2$, and WC samples. The fitting parameters show that the rms roughness at the $\mathrm{Co} / \mathrm{Mo}$ interfaces increases with the deposition of consecutive layers from 0.2 to $0.5 \mathrm{~nm}$. The thicknesses of the individual Co and Mo layers are collected in Table [. The thickness determined from the simulated XRR profiles deviates from the assumed nominal thickness of the component layers. The actual measured quantities are used in numerical simulations of magnetostatic and magnetodynamic properties discussed later in this work.

The crystalline structure of the component layers was also examined with XRD. Figure 3 shows a schematic configuration of the XRD measurements at different $\phi$ and $\psi$ angles. A change in the angle $\phi$ corresponds to a rotation of the sample around the $z$ axis, which is perpendicular to the sample plane, whereas the $\psi$ angle is a tilt of the sample around the $x$ axis. The black line with a dot on the sample defines the initial position of the sample for $\phi=0^{\circ}$. Figure 4 shows $\theta-2 \theta$ (at $\phi=0^{\circ}$ 


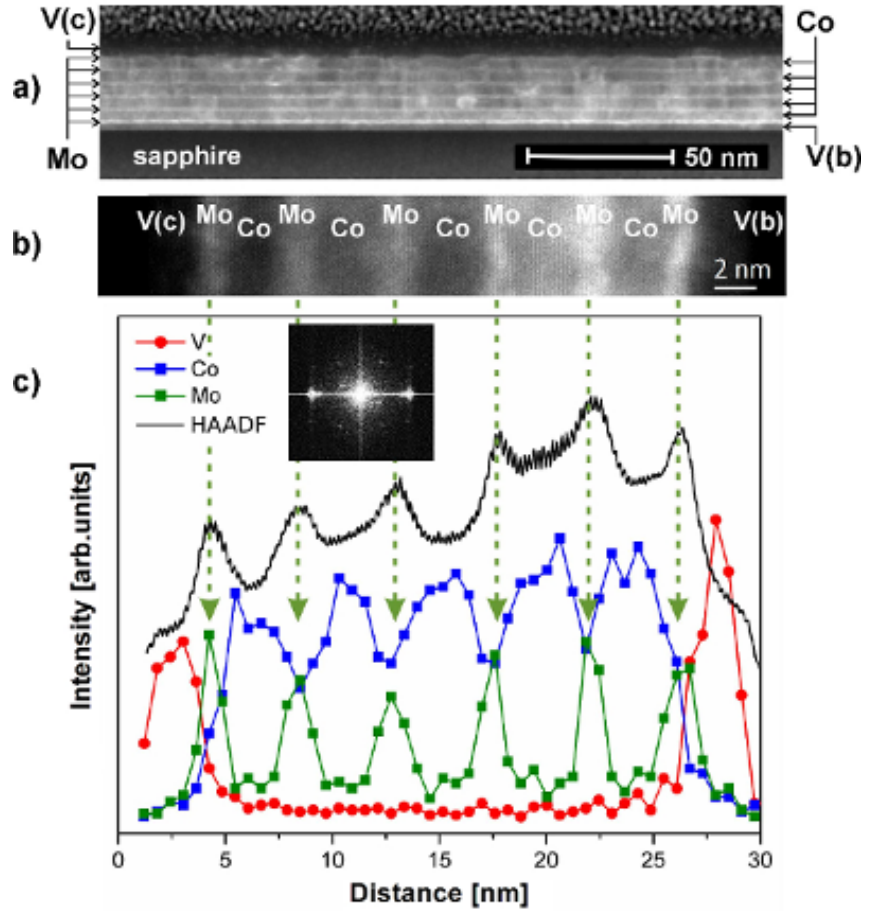

FIG. 1: TEM analysis of sample C2: (a) Low-magnification TEM image of Co/Mo multilayer. (b) High-resolution image with visible atomic layers with $\mathrm{V}$ buffer $[\mathrm{V}(\mathrm{b})]$ located on the right side. (c) In-depth EDX spectroscopy profile of chemistry of multilayer components: Co (blue), Mo (green), V (red), and high-angle annular dark-field section (black). The inset shows a Fourier transform across the whole multilayer shown in panel (b).

TABLE I: Thicknesses of component layers (in $\mathrm{nm}$ unless stated otherwise) obtained from XRR measurements.

\begin{tabular}{ccccc}
\hline \hline & Layer & $\mathrm{C} 1$ & $\mathrm{C} 2$ & WC \\
\hline Substrate: & $\mathrm{Al}_{2} \mathrm{O}_{3}$ & $0.6 \mathrm{~mm}$ & $0.6 \mathrm{~mm}$ & $0.6 \mathrm{~mm}$ \\
$\mathrm{~V}$ & 2.78 & 2.46 & 2.53 \\
$\mathrm{Mo}$ & 0.67 & 0.61 & 0.81 \\
$\mathrm{Co}$ & 2.14 & 3.34 & 1.48 \\
$\mathrm{Mo}$ & 0.52 & 0.77 & 0.89 \\
$\mathrm{Co}$ & 2.01 & 3.60 & 1.52 \\
$\mathrm{Mo}$ & 0.60 & 0.56 & 0.99 \\
$\mathrm{Co}$ & 2.03 & 3.70 & 1.03 \\
$\mathrm{Mo}$ & 0.55 & 0.57 & 0.89 \\
$\mathrm{Co}$ & 2.13 & 3.58 & 1.77 \\
$\mathrm{Mo}$ & 0.68 & 0.76 & 1.05 \\
$\mathrm{Co}$ & 2.21 & 3.20 & 1.75 \\
$\mathrm{Mo}$ & 0.64 & 0.68 & 0.52 \\
Capping: & $\mathrm{V}$ & 3.33 & 3.00 & 3.33 \\
\hline \hline
\end{tabular}

and $\psi=0^{\circ}$ ) XRD spectra from $\mathrm{C} 1, \mathrm{C} 2$, and $\mathrm{WC}$ samples. Additional peaks appear on the right side of the two very strong peaks that originate from the sapphire $\left(\mathrm{Al}_{2} \mathrm{O}_{3}\right)$ substrate. The arrows indicate the positions of peaks for

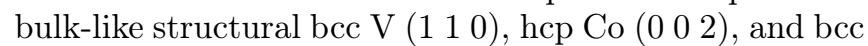
Mo ( $\left.\begin{array}{lll}1 & 1 & 0\end{array}\right)$ and their second-order peaks. However, their

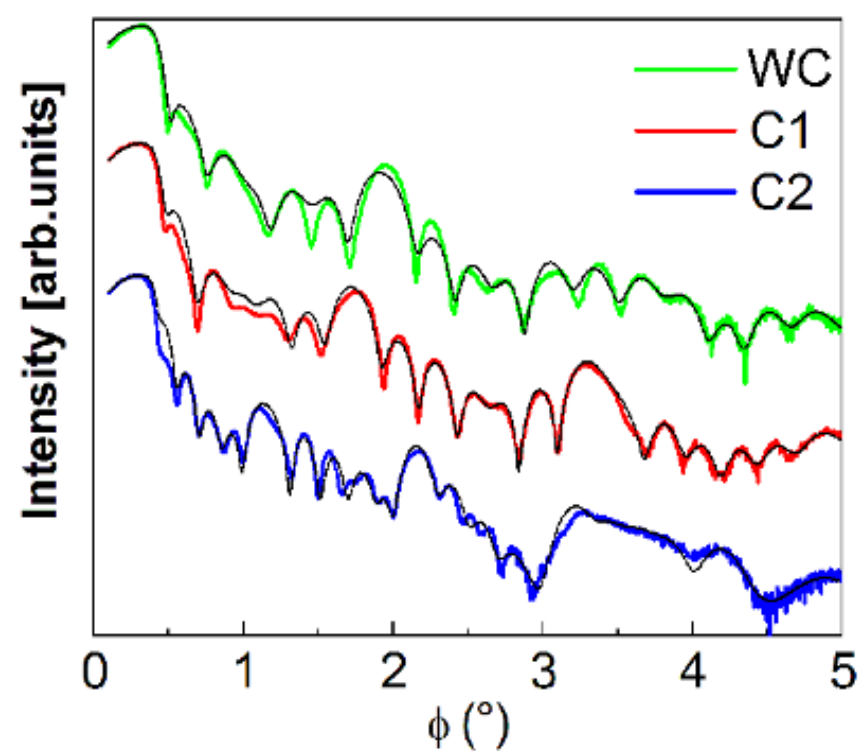

FIG. 2: XRR profiles of WC (green line), C1 (red line), and C2 (blue line) samples and corresponding fits (black lines).

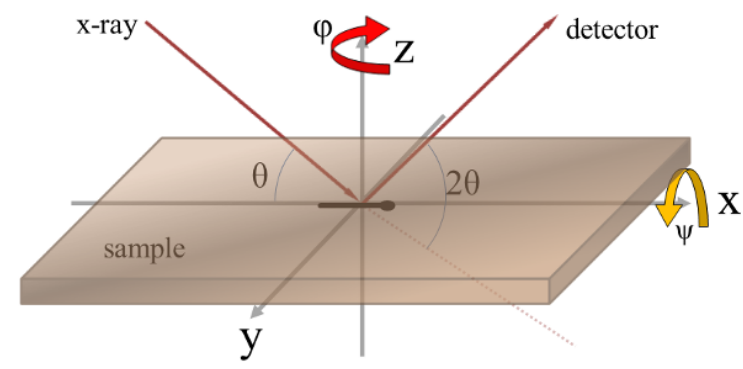

FIG. 3: Scheme of the XRD scans: $\phi$ (rotation around the $z$ axis) and $\psi$ (rotation around the $x$ axis). The black line with a dot on the sample indicates the sample's initial position $(\phi=0)$.

positions do not coincide with those of superlattice peaks in the measured diffraction profile. To explain the origin of these peaks, the $\theta-2 \theta$ diffraction model was used to simulate the periodic structure of the artificial superlattice [41. The sample structures were simulated by using the thicknesses of the individual layers obtained from the XRR measurements. The calculated $\theta-2 \theta$ diffraction profiles are plotted in Fig. 4 as red lines. The simulations show clearly that the peaks in the diffraction profiles are not structural peaks from Mo and Co layers but originate from the $\mathrm{Co} / \mathrm{Mo}$ superlattices and reproduce in entirety the experimental patterns. These results confirm the good planar growth of the layered structure throughout the stack.

Figure 5 shows $\phi$ scans for the $\mathrm{C} 1, \mathrm{C} 2$, and WC samples at $2 \theta=41.6^{\circ}$, which corresponds to hcp Co (10 0 ) planes. The angle $\psi$ was set to $87.2^{\circ}$ (i.e., near $90^{\circ}$ ), which corresponds to the angle between the hcp Co $(10$ $0)$ and $\left(\begin{array}{lll}0 & 0 & 2\end{array}\right)$ planes. The angle $87.2^{\circ}$ was chosen be- 

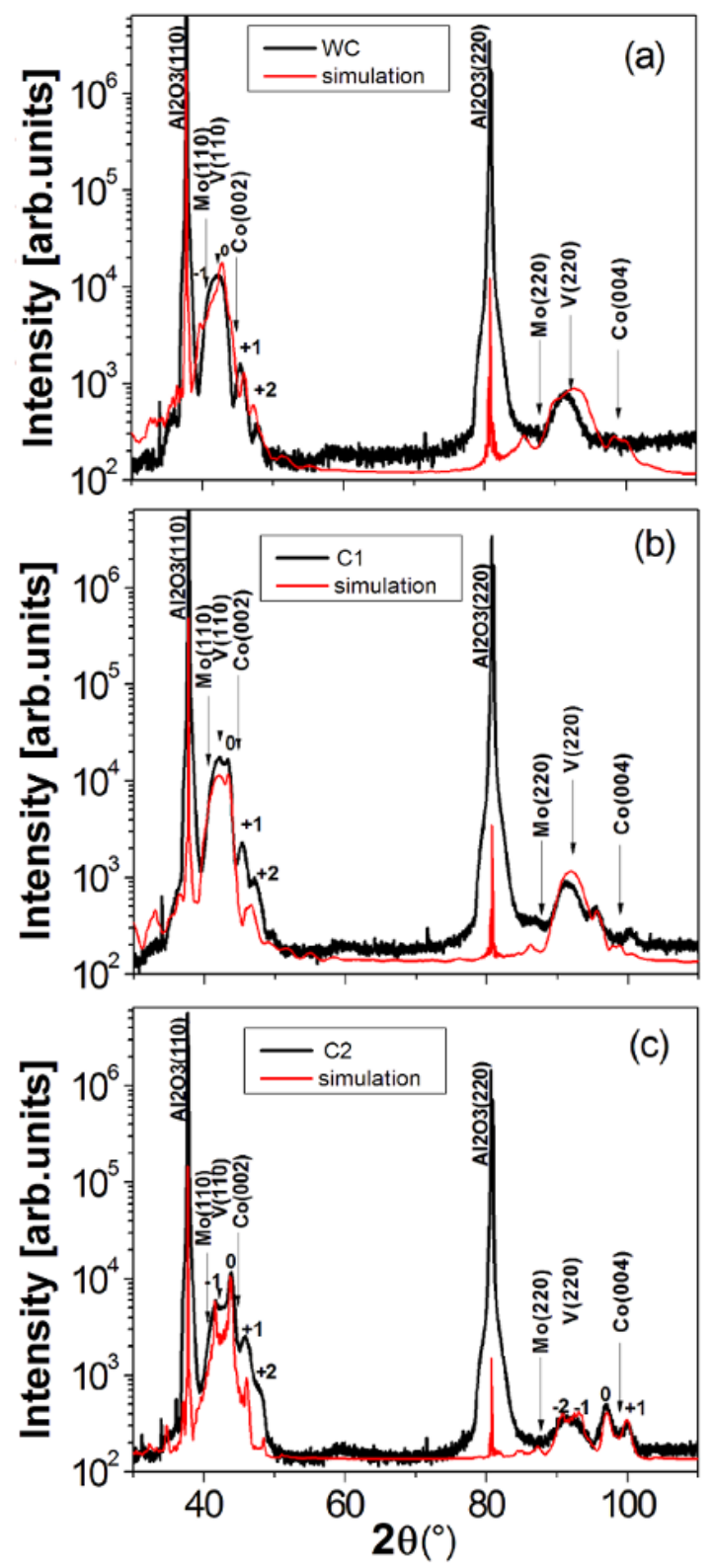

FIG. 4: XRD $\theta-2 \theta$ spectra (black lines) for multilayered samples WC (a), C1 (b), and C2 (c). Red lines are the simulation of the diffraction profiles.

cause, at higher angles, the peak intensities were strongly suppressed because the x-ray beam was partially blocked by the sample edge. The $\mathrm{C} 1$ and $\mathrm{C} 2$ samples exhibit six pronounced peaks from Co hcp ( $\left.\begin{array}{lll}1 & 1 & 0\end{array}\right)$ planes, whereas these peaks do not appear for the WC sample. The six peaks in the diffraction profiles originate from the sixfold symmetry of the Co hexagonal structure. Unlike the $\mathrm{C} 1$ and $\mathrm{C} 2$ structures, the WC multilayer does not have sixfold symmetry. The Co sublayers in the WC sample are the thinnest layers in comparison with the other samples. Due to lattice mismatch at the $\mathrm{Co} / \mathrm{Mo}$ interfaces, the Co layer in the initial growth stage has poor crystalline structure (most probably a mixture of hcp and fcc

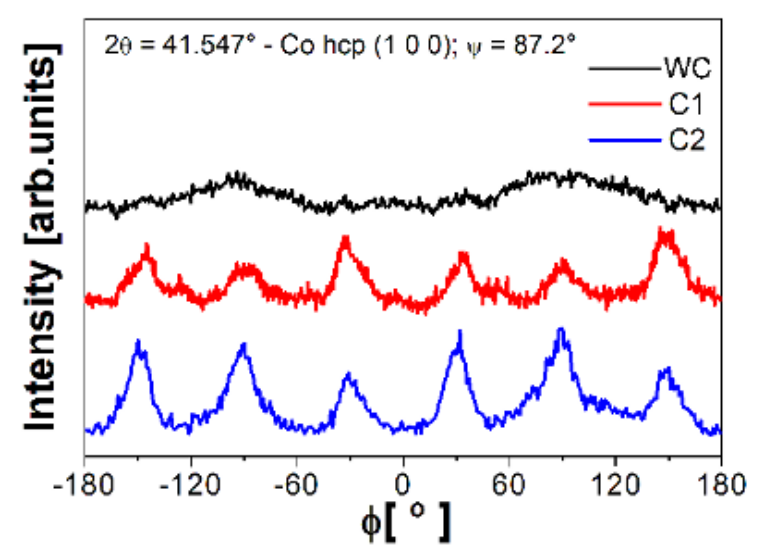

FIG. 5: XRD $\phi$ scans for multilayer samples C1, C2, and WC measured at $2 \theta=41.6^{\circ}$ and $\Psi=2 \theta=87.2^{\circ}$.

phases, with numerous stacking faults). In addition, the crystallinity of the Mo spacer, which affects the growth of the subsequent Co layer, depends on the crystalline structure of the Co film underneath.

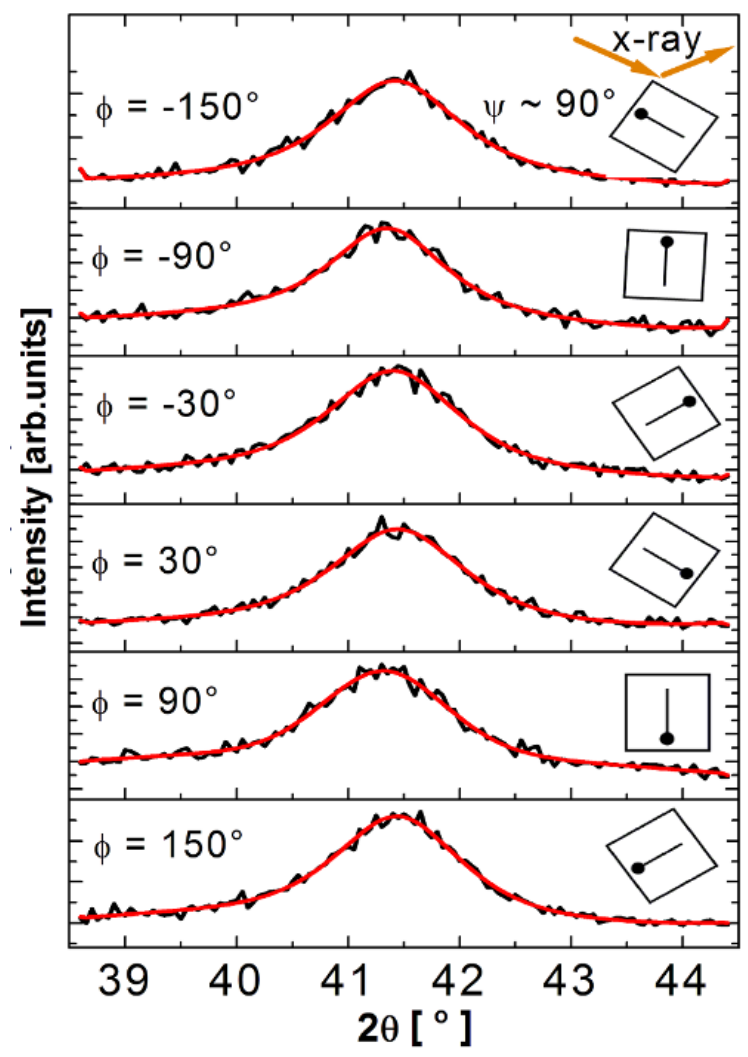

FIG. 6: XRD $\theta-2 \theta$ profiles (black lines) for C1 sample. Red lines are fits to the diffraction profiles.

Figure 6 shows $\theta-2 \theta$ scans of the $\mathrm{C} 1$ sample taken around $2 \theta \approx 41.6^{\circ}$, which corresponds to the Co hcp $\left(\begin{array}{lll}1 & 0 & 0\end{array}\right)$ planes. Measurements were taken at six maxima obtained from the $\phi$ scan (see Fig. 5) with the sample 
TABLE II: Angular $(\phi)$ dependence of $2 \theta$ and the calculated lattice parameters for $\mathrm{C} 1$ sample.

\begin{tabular}{|c|c|c|c|c|c|c|c|}
\hline \multicolumn{4}{|c|}{ Co $\left(\begin{array}{lll}1 & 1 & 0\end{array}\right)$} & \multicolumn{4}{|c|}{ Co $\left(\begin{array}{lll}1 & 0 & 0\end{array}\right)$} \\
\hline $\begin{array}{l}\phi \\
\left({ }^{\circ}\right) \\
\end{array}$ & $\begin{array}{l}2 \theta \\
\left({ }^{\circ}\right) \\
\end{array}$ & $\begin{array}{c}d(110) \\
(\mathrm{nm})\end{array}$ & $\begin{array}{c}a_{h k l} \\
(\mathrm{~nm})\end{array}$ & $\begin{array}{l}\phi \\
\left({ }^{\circ}\right)\end{array}$ & $\begin{array}{l}2 \theta \\
\left(^{\circ}\right)\end{array}$ & $\begin{array}{c}d(100) \\
(\mathrm{nm})\end{array}$ & $\begin{array}{c}a_{h k l} \\
(\mathrm{~nm})\end{array}$ \\
\hline-150 & 41.41 & 2.1787 & 2.5157 & -120 & 75.44 & 1.2590 & 2.5181 \\
\hline-90 & 41.33 & 2.1827 & 2.5204 & -60 & 75.40 & 1.2596 & 2.5192 \\
\hline-30 & 41.40 & 2.1792 & 2.5163 & 0 & 75.61 & 1.2566 & 2.5132 \\
\hline 30 & 41.42 & 2.1782 & 2.5151 & 60 & 75.41 & 1.2595 & 2.5189 \\
\hline 90 & 41.32 & 2.1832 & 2.5209 & 120 & 75.43 & 1.2591 & 2.5183 \\
\hline 150 & 41.42 & 2.1782 & 2.5151 & 180 & 75.57 & 1.2572 & 2.5144 \\
\hline
\end{tabular}

tilted by $\psi \approx 90^{\circ}$ about the $x$ axis. The insets in Fig. 6 show the $\phi$ angles of the sample during the measurement. The angular positions of the peaks were determined from the fits to the peak profiles (red lines). A similar analysis was made for the Co ( $\left(\begin{array}{lll}1 & 1 & 0\end{array}\right)$ peak. Additionally, from the peak positions for different $\phi$ angles, the interplanar distances of Co $\left(\begin{array}{lll}1 & 0 & 0\end{array}\right)$ and Co $\left(\begin{array}{lll}1 & 1 & 0\end{array}\right)$ were calculated, with the detailed results presented in Table III Figure 7(a) illustrates a Co hexagonal cell with the indicated interplanar spacings. Figures 7(b) and 7(c) show the interplanar spacings $d(100)$ and $d(110)$ in the respective directions. The interplanar spacing $d(100)$ is larger along the direction marked by the thick black pointer inside the cell than in the direction perpendicular to the pointer. Similarly, the distances $d(110)$ are considerably larger in the angular directions $\phi= \pm 60^{\circ}, \pm 120^{\circ}$ than at $\phi=0^{\circ}, 180^{\circ}$. These results show clearly that the hexagonal Co cells are stretched in the direction indicated by the black pointer. The same type of Co-lattice deformation was reported by Prokop et al. [42].

Figure 8 shows the lattice constant versus $\phi$ calculated from the interplanar spacings $d(100)$ and $d(110)$. The larger lattice constant is for $\phi=-90^{\circ}$ and $90^{\circ}$. Similar measurements (data not shown) were carried out for the $\mathrm{C} 2$ sample and revealed the same relations. How the determined in-plane distortion of the Co cell in the $\mathrm{C} 1$ and C2 samples affects the magnetic properties is discussed later in this work. The results suggest that uniaxial strain is present in the sample plane. Magnetoelastic coupling should give rise to magnetic in-plane anisotropy. As discussed in the next sections, such anisotropy is clearly developed and imposes an easy-axis orientation of $90^{\circ}$. The same relation between the lattice deformation and magnetic anisotropy was reported in Ref. 442.

\section{MAGNETIC STUDIES}

\section{A. Theoretical model}

This subsection presents the macrospin and micromagnetic models that allow us to calculate SAF magnetization hysteresis loops and to analyze the magnetic dynamics of the considered structure in terms of resonance (a)

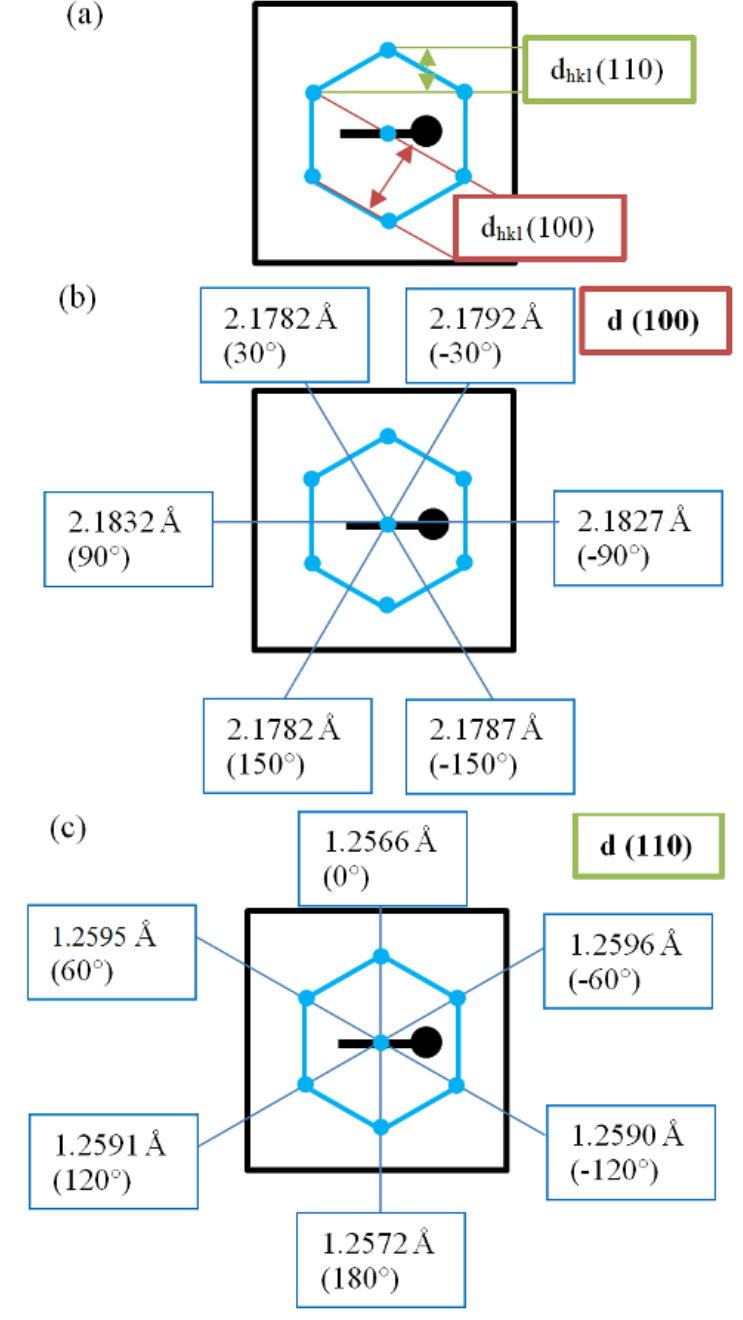

FIG. 7: Strain-induced deformation of the hexagonal Co cell in the C1 sample: (a) Definition of the interplanar distances, (b) azimuthal $(\phi)$ dependence of $d(100)$, and (c) $d(110)$ interplanar distances.

frequencies and resonance-mode intensities.

\section{Macrospin}

To effectively simulate the system, the macrospin approach was applied. The resonance frequencies of the magnetic structures can be easily calculated by using the well-established Smit-Beljers theory [43. However, here the proposed approach is more relevant to deal with superlattices consisting of several strongly coupled magnetic layers. The superlattice investigated in this paper consists of five coupled magnetic Co layers separated by nonmagnetic Mo layers of different thickness. We observe experimentally the magnetic response from the dynamic behavior of the magnetic moments of each layer, which are described by five pairs of spherical angles (polar $\theta_{i}$ 


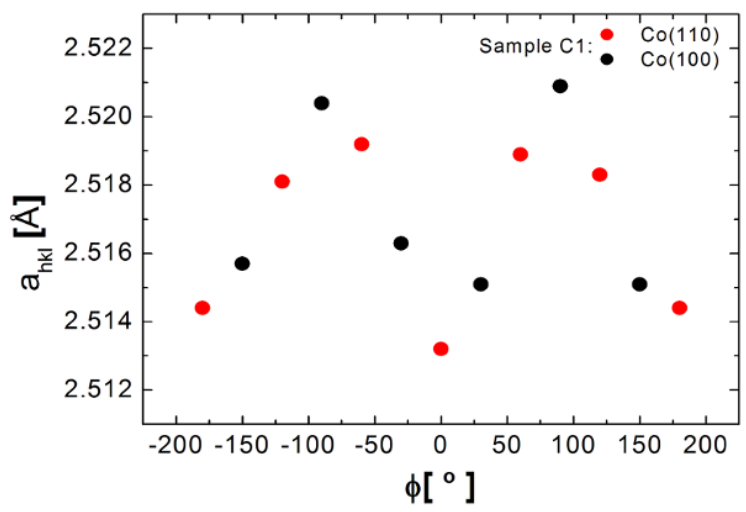

FIG. 8: Interplanar distances $a_{h k l}$ as a function of $\phi$ in Co hcp cell in C1 sample.

and azimuthal $\left.\phi_{i}\right)$ :

$$
M_{\boldsymbol{i}}=M_{S, i}\left[\sin \theta_{i} \cos \phi_{i}, \sin \theta_{i} \sin \phi_{i}, \cos \theta_{i}\right],
$$

where $i$ corresponds to the $i$ th cobalt layer. Therefore, the magnetization dynamics of the system is described by five coupled Landau-Lifshitz-Gilbert (LLG) equations,

$$
\frac{d \boldsymbol{M}_{\boldsymbol{i}}}{d t}=-\gamma_{e} \boldsymbol{M}_{\boldsymbol{i}} \times \boldsymbol{H}_{\mathrm{eff}, \mathrm{i}}+\frac{\alpha_{g}}{M_{S, i}} \boldsymbol{M}_{\boldsymbol{i}} \times \frac{d \boldsymbol{M}_{\boldsymbol{i}}}{d t},
$$

where $\gamma_{e} \approx 1.760859644 \times 10^{11} \frac{\mathrm{rad}}{\mathrm{sT}}$ is the gyromagnetic ratio, and $\alpha_{g}$ is the Gilbert damping parameter for each layer. The value of the damping parameter $\left(\alpha_{g}=0.02\right)$ was determined from the widths of VNA-FMR resonant peaks from the WC sample (an example of such spectra is discussed in Sec. VC). In general, the damping in the Co layer may vary considerably, even by one order of magnitude [4], depending on the thickness of the Co and adjacent Mo layers. However, a smaller variation in $\alpha_{g}$ has been reported for thicker layers. In our case, both Co and Mo layers are sufficiently thick to fix the value of $\alpha_{g}$ for all samples considered herein without harming the reliability of the simulations. The effective fields can be expressed as follows:

$$
\boldsymbol{H}_{\mathrm{eff}, \mathrm{i}}=-\nabla_{\theta_{i}, \phi_{i}} U,
$$

where $\nabla_{\theta_{i}, \phi_{i}}$ are the relevant gradients in spherical coordinates. The total magnetic energy density $U$ can be written as

$$
\begin{aligned}
U\left(\theta_{i}, \phi_{i}\right)= & \sum_{i j}-K_{i} d_{C o_{i}}\left(\cos \phi_{i} \sin \theta_{i}\right)^{2}-d_{C o_{i}} \boldsymbol{M}_{\boldsymbol{i}} \cdot \boldsymbol{H}_{\mathrm{ext}} \\
& -d_{C o_{i}} \boldsymbol{M}_{\boldsymbol{i}} \cdot \boldsymbol{H}_{d e m, i}-J_{i j} \boldsymbol{M}_{\boldsymbol{i}} \cdot \boldsymbol{M}_{\boldsymbol{j}}
\end{aligned}
$$

where $K_{i}$ is the anisotropy constant of the $i$ th Co layer, $\boldsymbol{H}_{\text {ext }}$ is an external magnetic field, $\boldsymbol{H}_{\mathrm{dem}, i}$ is the demagnetizing field within the layers, and $J_{i j}$ is the bilinear interlayer exchange-coupling energy constant.

To calculate the resonance frequencies, we assume small-amplitude oscillations, which allows us to linearize
Eq. 22 around the energy minima at given external magnetic-field magnitudes. Provided small-amplitude oscillations are taken into account, the angular solutions of Eq. 22 can be expressed as harmonic oscillations of magnetization angles in polar coordinates:

$$
\begin{aligned}
\theta_{i}(t) & =\theta_{0, i}+\delta \theta_{i}(t)=\theta_{0, i}+\delta \theta_{i} e^{i\left(\omega_{i} t\right)} \\
\phi_{i}(t) & =\phi_{0_{i}}+\delta \phi_{i}(t)=\phi_{0, i}+\delta \phi_{i} e^{i\left(\omega_{i} t\right)}
\end{aligned}
$$

where $\left(\theta_{0, i}, \phi_{0, i}\right)$ describe the equilibrium orientation of the magnetization in all magnetic layers (in the absence of a driving radio frequency magnetic field). In general, the amplitudes $\left(\delta \theta_{i}, \delta \phi_{i}\right)$ are complex and include a phase shift between the magnetization and the timedependent driving force (e.g., an $\mathrm{AC}$ external magnetic field). Also, the frequencies $\omega_{i}$ are complex. Their real parts $\omega_{R, i}$ correspond to resonance angular frequencies, whereas the imaginary parts $\frac{1}{\omega_{I, i}}$ give the half-life of decaying free oscillations due to presence of effective damping. As done in Ref. [45, Eq. 22 can be rewritten in matrix form as

$$
\dot{\boldsymbol{\alpha}}=\boldsymbol{v}\left(\theta_{i}, \phi_{i}\right)^{T}
$$

where $\boldsymbol{\alpha}=\left(\alpha_{1}, \ldots, \alpha_{10}\right)^{T} \equiv\left(\theta_{1}, \phi_{1}, \ldots, \theta_{5}, \phi_{5}\right)^{T}$ is a 10 element column vector consisting of time derivatives of spherical angles given by Eq. (6). Note that components of $\boldsymbol{\alpha}$ are indexed by $k$ varying from 1 to 10 . Thus, the LLG equation in polar coordinates has the general form

$$
\dot{\boldsymbol{\alpha}}=\boldsymbol{v}\left(\theta_{i}, \phi_{i}\right)^{T},
$$

where $\boldsymbol{v}$ is the right-hand-side (RHS) vector of the LLG equation. After linearization of $\boldsymbol{v}$ with respect to small deviations in $\theta_{i}$ and $\phi_{i}$ from their stationary values, one can write Eq. (8) in the form

$$
\dot{\boldsymbol{\alpha}}=\hat{X} \boldsymbol{\Gamma}(t)
$$

where $\hat{X}$ is a $10 \times 10$ matrix consisting of the derivatives of the RHS of Eq. (8) with respect to the angles $\theta_{i}, \phi_{i}$ (i.e., $X_{k j} \equiv \frac{\partial v_{k}}{\partial \alpha_{j}}$ ), while $\boldsymbol{\Gamma}(t)=\left(\delta \alpha_{1}(t), \ldots, \delta \alpha_{10}(t)\right)^{T}$ is a vector containing time-dependent angle differentials [i.e., $\delta \alpha_{1}(t) \equiv \delta \theta_{1}(t), \delta \alpha_{2}(t) \equiv \delta \phi_{1}(t)$, etc.], as defined in Eq. (6). Next, Eq. (9) can be rewritten as an eigenvalue problem of the matrix $\hat{X}$ :

$$
|\hat{X}-\omega \hat{I}|=0 .
$$

The eigenvalues $\omega_{i}$ determine five distinguished resonance (natural) angular frequencies of the system, $\omega_{R, k}=$ $\operatorname{Re} \omega_{i}$. On the other hand, the general solution of Eq. (2) can be expressed by using a linear combination of eigenvectors corresponding to all eigenvalues $\omega$ in the following form:

$$
\boldsymbol{\alpha}(t)=\sum_{k=0}^{10} c_{k} \mathbf{v}_{\boldsymbol{k}} e^{i \omega_{k} t},
$$


where $\mathbf{v}_{k}$ is a 10-element eigenvector corresponding to the eigenvalue $\omega_{k}$, and $c_{k}$ are the scalar coefficients that can be combined as one column vector $c$. Equation (11) describes the free damped oscillations that may occur when the magnetizations are initially tilted away from their minimum energy points by, for example, an external magnetic field. For $t=0$, Eq. 111) can be expressed by using the scalar product

$$
\boldsymbol{\alpha}(0)=\boldsymbol{c} \cdot \mathbf{v},
$$

so that the columns of the vector $\mathbf{v}$ are the eigenvectors $\mathbf{v}_{k}$. Thus, the components of $\boldsymbol{c}$ can be derived by using the initial conditions for magnetization angles and their time derivatives; namely,

$$
\boldsymbol{\alpha}(0)=\sum_{k=0}^{10} c_{k} \mathbf{v}_{\boldsymbol{k}}=\boldsymbol{\alpha}_{\mathbf{0}}
$$

and

$$
\dot{\boldsymbol{\alpha}}(0)=\sum_{k=0}^{10} i \omega_{k} c_{k} \mathbf{v}_{\boldsymbol{k}}=\boldsymbol{v}\left(\boldsymbol{\alpha}_{\mathbf{0}}\right)
$$

where $\boldsymbol{\alpha}_{0} \equiv\left(\alpha_{1}, \ldots, \alpha_{10}\right)=\left(\theta_{0,1}, \phi_{0,1}, \ldots, \theta_{0,5}, \phi_{0,5}\right)$. By combining Eqs. (13) and (14), the formula for the modecoefficient vector $\boldsymbol{c}$ takes the form

$$
\boldsymbol{c}=\boldsymbol{v}\left(\boldsymbol{\alpha}_{0}\right) \cdot \tilde{\mathbf{v}}^{-1},
$$

where $\tilde{\mathrm{v}}_{k} \equiv\left(1+i \omega_{k}\right) \mathrm{v}_{k}$. The initial values of angles $\boldsymbol{\alpha}_{0}$ can be determined by minimizing the total magnetic energy (4). The intensity $\mathcal{I}_{k}$ of the given resonance mode $\left(\omega_{k}\right)$ is defined as

$$
\mathcal{I}_{k} \equiv \max (\xi(t))-\min (\xi(t)),
$$

where

$$
\begin{aligned}
\xi(t)= & \sum_{l=1}^{5} \boldsymbol{M}_{\mathbf{0}, \boldsymbol{l}}+\Delta \boldsymbol{M}_{\boldsymbol{l}} \\
\approx & \sum_{l=1}^{5} M_{S, l}\left\{\cos \left[\alpha_{0,2 l}+\operatorname{Re}\left(c_{k, 2 l} \cdot \mathrm{v}_{\mathrm{k}, 2 \mathrm{l}} \mathrm{e}^{\mathrm{i} \omega_{\mathrm{k}} \mathrm{t}}\right)\right]\right. \\
& \left.+\sin \left[\alpha_{0,2 l}+\operatorname{Re}\left(c_{k, 2 l} \cdot \mathrm{v}_{\mathrm{k}, 21} \mathrm{e}^{\mathrm{i} \omega_{\mathrm{k}} \mathrm{t}}\right)\right]\right\}
\end{aligned}
$$

The definition only accounts for the changes in the inplane magnetization (described by $\phi_{i}$ ). However, it is clearly consistent with the fast Fourier transform procedure that we apply to the results of the micromagnetic simulation. Equation (17) ensures that $\mathcal{I}_{k}$ is suppressed when, for example, adjacent antiparallel magnetization vectors oscillate both with the same phase. On the contrary, $\mathcal{I}_{k}$ is enhanced when both antiparallel magnetizations oscillate with the opposite phases.

\section{Micromagnetics}

Equation (16) allows us to compare macrospinresonance-mode intensities with the micromagnetic ones. Thus we have performed micromagnetic simulations with use of the OOMMF package [40]. First, we modeled the hysteresis loops, then we calculated the dynamic magnetization response of the structure upon excitation by an external field. The latter gave us the resonance frequencies of the system and the intensities of the resonance modes. Both static and dynamic micromagnetic simulations employ cuboid-shaped simulation cells with dimensions $5 \mathrm{~nm} \times 5 \mathrm{~nm} \times 1.5 \mathrm{~nm}$. The exchange constant for all Co layers was taken as $A_{\mathrm{ex}}=3 \times 10^{-11} \mathrm{~J} / \mathrm{m}$. The dynamics simulation scheme is similar to that used in our previous works [45, 46]: it starts from the state of fully relaxed magnetization of the $\mathrm{Co} / \mathrm{Mo}$ superlattice. Next, to excite a dynamic response, a short magnetic-field pulse (of the order of $10 \mathrm{Oe}$ ) is applied, and the magnetization response of the system is processed by using a fast Fourier transform to disclose the natural frequencies at a given external magnetic field. As shown in Secs. VB and $\mathrm{VC}$, the micromagnetic simulations fully confirm the reliability of the macrospin model in both cases: coupled and WC Co/Mo multilayers. Next, these two models are applied to comprehensively characterize coupled and WC $\mathrm{Co} /$ Mo superlattices.

\section{B. Magnetostatic characterization}

This subsection presents the experimental results from $\operatorname{VSM}[M(H)]$ and MR $[M R(H)]$ measurements of the three SAF structures considered (i.e., samples C1, C2, and $\mathrm{WC})$. To reproduce the observed relations and to determine the magnetic parameters, the macrospin model is fit to the experimental data. In particular, we estimate the magnitude of the coupling energy between Co layers through a Mo spacer. Next, the reversal processes in SAF are presented and analyzed through macrospin modeling. The micromagnetic simulations show the reliability of this macrospin approach.

\section{Hysteresis loops and magnetoresistance}

To gain insight into the magnetization-reversal process in the structures considered, VSM and MR measurements were carried out. The hysteresis loops and MR dependencies were measured in the magnetic field applied in the sample planes along mutually orthogonal easy and hard axes being a consequence of magnetoelastic strain of the hep cobalt cell induced at the $\mathrm{Co} / \mathrm{Mo}$ interfaces (this effect is discussed in Sec. IV and in Ref. [35]). The MR in Co/Mo multilayers usually takes very small values and does not exceed $0.1 \%$ regardless of the presence of relatively strong interlayer coupling [39, 47. However, its magnetic-field dependence precisely reflects 


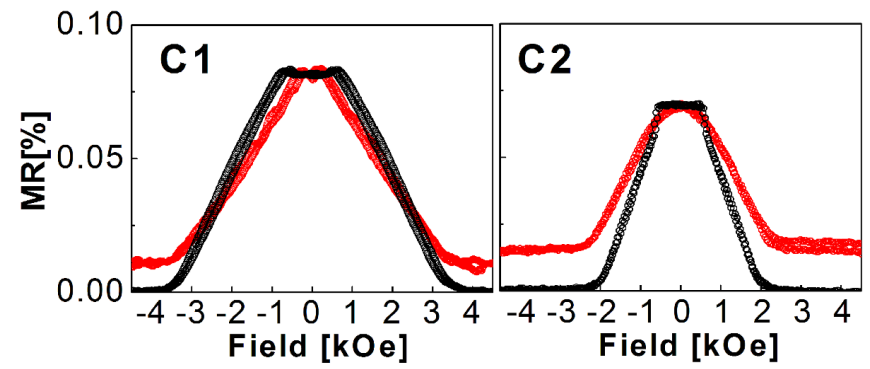

FIG. 9: Magnetoresistance of samples with AFM-coupled magnetic layers: C1 (left panel) and C2 (right panel) measured with magnetic field applied along the hard axis (red points) and easy axis (black points).

all the magnetic features revealed by the $M(H)$ hysteresis loops. The $M R(H)$ data turn out to be useful to determine the magnetic parameters of the SAF structures. The dominating contribution to the total MR is related to the GMR effect, which is very sensitive to the relative angle between the magnetizations of two neighboring magnetic layers. Therefore, simultaneously fitting the macrospin model to the $M(H)$ and $M R(H)$ dependencies should result in optimal and reliable macrospin magnetic parameters. However, this approach can be applied only to the $\mathrm{C} 1$ and $\mathrm{C} 2$ samples; the MR is completely suppressed in the WC sample, which may be related to the thickness of the Co layers [48] being less than those of other samples. For this reason, for the WC sample, only VSM data are used for the model fitting.

To model the experimental data presented in this paper, we used a self-consisting fitting of the macrospin model to the VSM hysteresis loops and MR relations in both the easy and hard directions. The orientation of the magnetic moments was calculated by minimizing the total magnetic energy of the system given by Eq. (4). In the fitting procedure, we used the Co layers thicknesses determined by XRR measurements. The GMR value was calculated by using the standard formula $R_{i j}\left(\theta_{i j}\right)=$ $R_{0}+\frac{\Delta R}{2}\left(\cos \theta_{i j}-1\right)$, where $\Delta R \equiv R_{A P, i j}-R_{P, i j}$ is the difference in resistance between layers $i$ and $j$ in the parallel (P) or antiparallel (AP) state, and $\theta_{i j}$ is the relative angle between magnetic (Co) layers $i$ and $j$ and depends implicitly on the external magnetic field $H$. In the current-in-plane configuration, the MR originates from each pair of magnetic layers, so the overall GMR of the whole structure is calculated by averaging $R_{i j}$ over all pairs at a given magnetic field [i.e., $R(H)=\frac{R_{i j}}{4}$ ]. Despite this simplification, the theoretical curves are sufficiently consistent with the experimental curves. The optimal set of macrospin magnetic parameters determined from the VSM and MR data is listed in Table III.

Figures 9 and 10 show the experimental and theoretical $M R(H)$ dependencies measured in samples $\mathrm{C} 1$ and $\mathrm{C} 2$ and the macrospin curves, and Fig. 11 shows the $M(H)$ relations, together with a detailed illustration of SAF magnetization reversal processes.
TABLE III: Set of macrospin magnetic parameters for C1, C2, and WC samples. The parameters are used in the theoretical plots of hysteresis loops and MR dependencies shown in Figs. 10,12

\begin{tabular}{|c|c|c|c|c|c|}
\hline Sample & Layer & $\overline{d_{i}[\mathrm{~nm}]}$ & $\overline{K_{i}\left[\frac{\mathrm{kJ}}{\mathrm{m}^{3}}\right]}$ & $M_{S, i}[\mathrm{~T}]$ & $\overline{J_{i, j}\left[\frac{\mathrm{mJ}}{\mathrm{m}^{2}}\right]}$ \\
\hline \multirow[t]{9}{*}{ C1 } & Co 1 & 2.14 & 1.0 & 1.35 & \multirow{3}{*}{-0.10} \\
\hline & Mo & 0.52 & & & \\
\hline & Co 2 & 2.01 & 1.0 & 1.34 & \\
\hline & Mo & 0.60 & & & \multirow[t]{2}{*}{-0.18} \\
\hline & Co 3 & 2.03 & 1.0 & 1.34 & \\
\hline & Mo & 0.55 & & & \multirow[t]{2}{*}{-0.20} \\
\hline & Co 4 & 2.10 & 1.0 & 1.35 & \\
\hline & Mo & 0.70 & & & \multirow[t]{2}{*}{-0.16} \\
\hline & Co 5 & 2.21 & 1.0 & 1.36 & \\
\hline \multirow[t]{9}{*}{ C2 } & Co 1 & 3.34 & 2.5 & 1.40 & \multirow{3}{*}{-0.15} \\
\hline & Mo & 0.77 & & & \\
\hline & Co 2 & 3.60 & 2.5 & 1.40 & \\
\hline & Mo & 0.56 & & & \multirow[t]{2}{*}{-0.21} \\
\hline & Co 3 & 3.70 & 2.5 & 1.40 & \\
\hline & Mo & 0.57 & & & \multirow[t]{2}{*}{-0.22} \\
\hline & Co 4 & 3.58 & 2.5 & 1.40 & \\
\hline & Mo & 0.76 & & & \multirow[t]{2}{*}{-0.16} \\
\hline & Co 5 & 3.20 & 2.5 & 1.40 & \\
\hline \multirow[t]{9}{*}{$\mathbf{W C}$} & Co 1 & 1.48 & 0 & 1.30 & \multirow{3}{*}{-0.012} \\
\hline & Mo & 0.86 & & & \\
\hline & Co 2 & 1.52 & 0 & 1.30 & \\
\hline & Mo & 0.99 & & & \multirow[t]{2}{*}{-0.012} \\
\hline & Co 3 & 1.03 & 0 & 1.10 & \\
\hline & Mo & 0.89 & & & \multirow[t]{2}{*}{-0.015} \\
\hline & Co 4 & 1.77 & 0 & 1.37 & \\
\hline & Mo & 1.05 & & & \multirow{2}{*}{-0.012} \\
\hline & Co 5 & 1.75 & 0 & 1.37 & \\
\hline
\end{tabular}

In addition to the contribution from the GMR, the magnetization orientation with respect to the current flow (anisotropic magnetoresistance - AMR) affects the resulting MR signal. In particular, the difference in MR measured along the easy and hard axes at saturation magnetic field, shown in Fig. 9, is the contribution from the AMR. The AMR increases with the thickness of the FM layers [4], so in sample C2 it is slightly greater than in sample $\mathrm{C} 1$. In the field along the easy axis, the MR attains a maximum value in a certain field range [Figs. 10(b) and 10(d)] corresponding to the width of the central hysteresis loop in $M(H)$ curves [Figs. 11(a) and 11(c)]. This is related to the stable AFM alignment of the magnetization in the component Co layers and the enhanced spin-sensitive scattering of electrons. The decrease in MR with magnetic field corresponds to a rotation of the magnetization toward saturation, where MR reaches its minimum value. The sloped $R(H)$ dependencies with the apex at $H=0$ in the field applied along the hard axis [Fig. 10(a) and 10(c)] correlates with the $M(H)$ curves that makes a loopless, tilted linear-like shape in Figs. 11(b) and 11(d). Such a reversible change of magnetization is expected when the magnetization of the Co component layers gradually rotates from the hard-axis direction (forced by the applied field) toward the orthogonal direction (i.e., easy axis), where the magnetization 

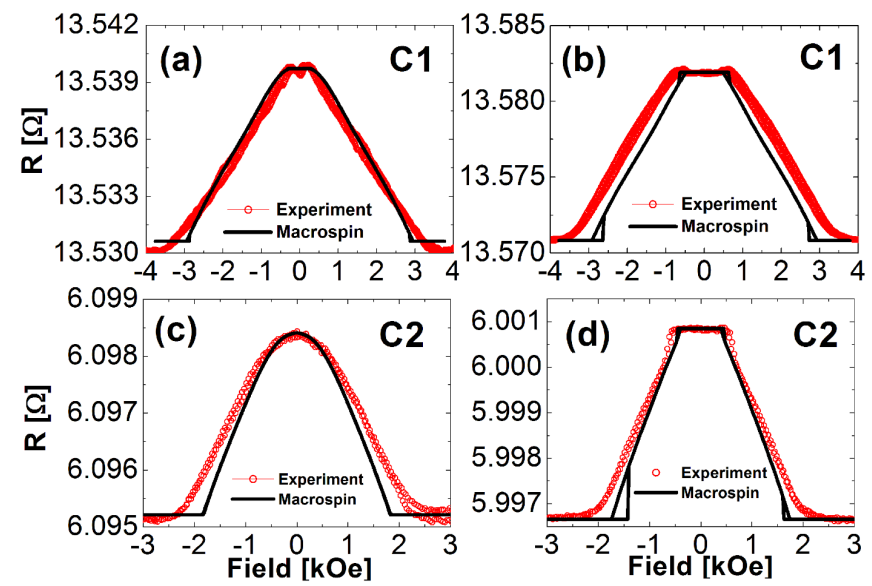

FIG. 10: Macrospin MR curves (black) fit to the experimental points (red) with a magnetic field applied along the hard axes of samples (a) C1 and (c) C2 or easy axes of samples (b) C1 and (d) C2.

is stable in the remanent state.

The experimental VSM relations measured in the $\mathrm{C} 1$ and C2 samples with the magnetic field applied along the easy axis exhibit a typical loop in the center of the $M(H)$ curve [Figs. 11 (a) and 11(c)]. Upon decreasing the magnetic field, the magnetizations of the two inner Co layers rotate coherently and gradually from parallel alignment (saturated state) to AFM ordering. The loop in the central part of the $M(H)$ dependence is a consequence of antiparallel magnetization configuration in the stack composed of an odd number of magnetic layers. This configuration is stable in a certain field range corresponding to the width of the hysteresis loop. A simulation of the macrospin precisely reproduces the central hysteresis loop. Two stable AFM orderings occur within the loop region ( 3 of 5 or 2 of 5 Co-layer magnetizations aligned along the external field), so that a transition between these orderings may occur. This is seen as an abrupt switching of magnetization in individual layers [compare magnetization alignment at 0 and $0.46 \mathrm{kOe}$ in Fig. [11(e)]. Moreover, the modeling reveals another narrow subloop existing at higher fields, close to the saturation of the SAF magnetization [see inset of Fig. 11(c)]. In these two regions there are two energy minima that are close to each other and separated by a low potentialenergy barrier. As a consequence, there are two possible magnetization configurations in these narrow ranges of magnetic field. This feature is clearly illustrated in Fig. 11(e). In the field range corresponding to the subloops, the magnetization alignment depends on the magnetic history of the multilayer: at $-1.4 \mathrm{kOe}$ (evolution from saturation towards AFM ordering), magnetization of the component layers is noncolinear, whereas it becomes fully colinear at $+1.4 \mathrm{kOe}$ (approaching saturation). Since the effect has been accounted for by the monodomain macrospin model, it can be related to the interlayer exchange coupling present in samples $\mathrm{C} 1$ and $\mathrm{C} 2$ and, to a lesser extent, to possible magnetization inhomogeneities and domain-wall pinning within the Co layers. Figure 12 shows the more realistic micromagnetic simulation of the hysteresis loop measured in sample $\mathrm{C} 2$ with the magnetic field applied along its easy axis (cf. Sec. VA 2). Similarly to the macrospin simulations, the micromagnetics exhibits small $M(H)$ subloops in the vicinity of the saturation field.

The fits obtained in both macrospin and micromagnetic approaches are satisfactory. Therefore, the effect of the interlayer coupling on the VSM relations dominates over the effects related to the complex magnetization distribution within the magnetic layers.

For the WC sample, very narrow hysteresis loops appear in both orthogonal magnetic-field directions applied in the sample plane [Fig. 11(g)]. This is evidence that the WC sample is isotropic. In addition, the interlayer coupling strength is not sufficiently high to produce a considerable hysteresis loop. The magnetization reversal is illustrated in Fig. 11(f). The magnetization rotates in all magnetic layers gradually in almost the entire range of magnetic field. The only possible switching-like event is at $H_{\text {ext }} \approx 0$ due to nonzero interlayer coupling. Moreover, the rotation of Co magnetization differs from that in the $\mathrm{C} 2$ sample. In particular, the central Co layer behaves differently than the others due to a smaller $M_{S}$ [35]. The magnetization of the middle layer is softer because of its perpendicular orientation with respect to the other Co layers at relatively high magnetic field [see Fig. 11 (f) at $H=0.25 \mathrm{kOe}$.

The difference in the anisotropic properties of samples $\mathrm{C} 1$ and $\mathrm{C} 2$ and the isotropic properties of sample $\mathrm{WC}$ can be explained in terms of strains induced in the component Co layers due to lattice mismatch at the $\mathrm{Mo} / \mathrm{Co}$ interfaces. A detailed discussion of this issue is provided in Sec. IV

\section{Ferromagnetic-resonance characterization}

This section discusses the magnetic dynamics in the coupled samples versus the WC sample. By using the VNA-FMR technique, we acquired the resonance response spectra from all samples. Next, the relations $f\left(H_{\text {res }}\right)$ are extracted from the VNA spectra by fitting the relevant resonance curves. In addition, the mode intensities are modeled by using the macrospin model and micromagnetics with the same magnetic parameters as used in Sec. VB1. Again, the reliability of the macrospin modeling is confirmed by comparing the results obtained from both approaches. The qualitative difference in $f\left(H_{\text {res }}\right)$ between coupled and WC samples is revealed and discussed. The detailed analysis of the optical and acoustic modes and their dependence on the external magnetic field in the coupled sample C2 is also provided.

Figure 13 shows the theoretical $f\left(H_{\text {res }}\right)$ relations and mode intensities predicted for sample $\mathrm{C} 2$ by Eqs. 10 

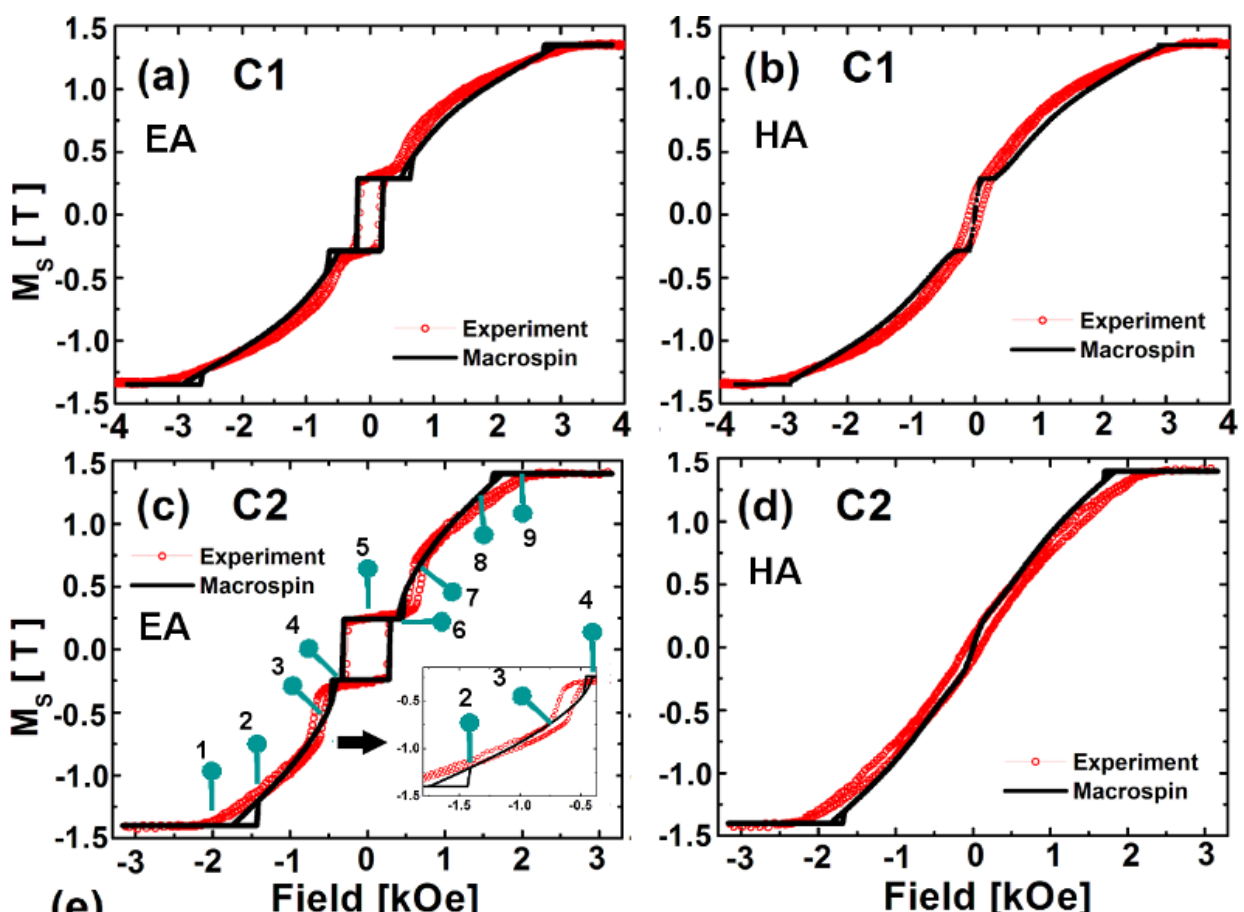

(e)

Field [kOe]

Field [kOe]
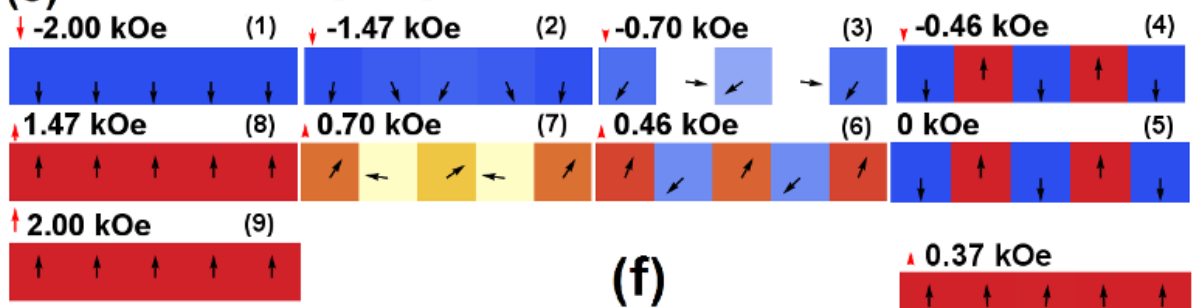

(f)
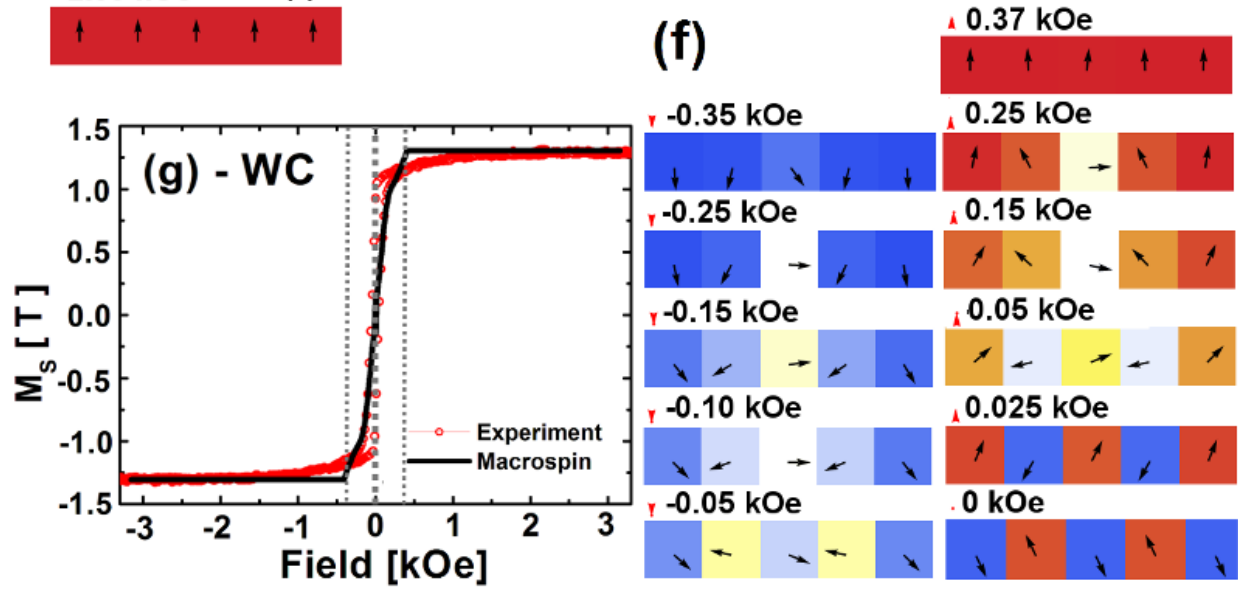

FIG. 11: Macrospin $M(H)$ curves fit to the experimental dependencies measured with the magnetic field applied along the easy and hard axes of samples (a), (b) C1, (c), (d) C2, and of (g) the isotropic WC sample. The easy-axis magnetization reversal process of (e) C2 and (f) WC samples as a function of external magnetic field is illustrated by the black arrows, which indicate the magnetization direction within each Co sublayer, and the related colors: from dark blue (downward magnetization) to red (upward magnetization). The blue thumbtack-shaped marks and the numbers on (c) the $M(H)$-C2 plot refer to (e) the respective magnetization configurations, whereas the vertical lines on $M(H)$ plot for the $(\mathrm{g})$ WC sample indicate the magnetic fields where (f) the magnetization reversal occurs in the WC SAF.

and (16), respectively, using the parameters from Table III.

The C2 sample exhibits a complex spectrum of resonance modes. However, the calculated modes are influenced rather by the interlayer coupling than by the magnetization inhomogeneity within Co layers. Similar complex FMR modes have already been observed; for example, in $\mathrm{Fe} / \mathrm{Cr}$ superlattices with bi-quadratic interlayer coupling [50] and, recently, in permalloy/Ru multilayers [13]. The macrospin prediction was compared with that 


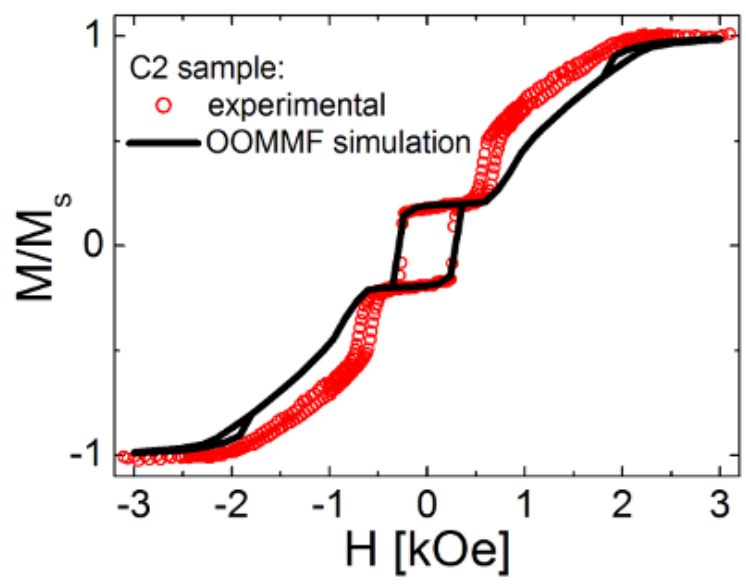

FIG. 12: Micromagnetically (OOMMF) simulated $M(H)$ curve versus experimental points obtained for sample C2. See Sec. VA2 for details.

calculated micromagnetically (cf. Sec. V A 2). The comparison is shown in Fig. 13 .
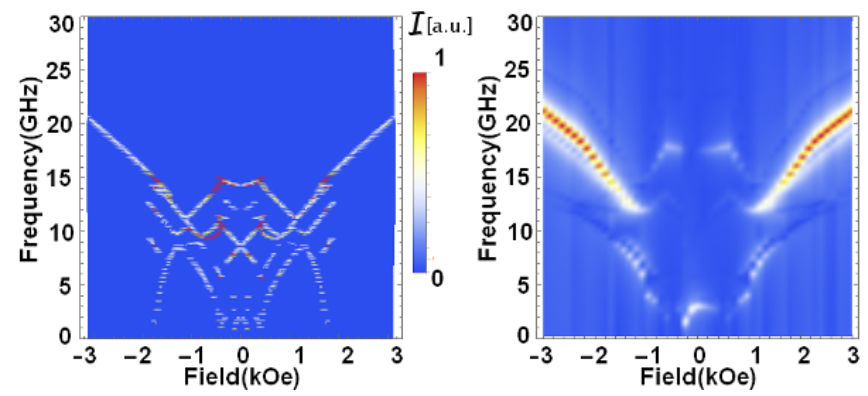

FIG. 13: Intensities of resonance modes $(\mathcal{I})$ calculated from Eq. 16 as a function of the resonance field $H_{\text {res }}$ and frequency $f$ with macrospin (left panel) and micromagnetic (right panel) approach for sample $\mathrm{C} 2$ with a magnetic field applied in the easy axis. The macrospin magnetic parameters are listed in Table III.

The micromagnetic and macrospin models agree well, particularly the resonance modes, which exhibit the same eigenfrequencies at high fields in both approaches. On the other hand, at low fields, the nontrivial dependence of resonance modes is accounted for by both the macrospin model and micromagnetics. The rich dispersion relation is due only to interlayer coupling, which is justified because comparing the micromagnetic hysteresis with the macrospin hysteresis does not reveal a significant qualitative difference [cf. Eq. [12)]. Nevertheless, the mode intensities differ in both approaches (i.e., low-field modes are more visible in macrospin than in micromagnetics). Similarly, the micromagnetic high-frequency mode at low field exhibits higher frequencies than its macrospin counterpart. A different number of resonance modes at low fields may be related to small amplitudes of micromagnetic oscillations. The barely visible white streaks at low resonance fields strongly suggest that such small ampli- tude micromagnetic oscillations are present. Therefore, we conclude that both approaches describe the resonance modes in the same qualitative way and the multiple resonance modes are related rather to collective magnetization dynamics of five Co exchange-coupled layers than to the magnetization inhomogeneities within them. Also, it proves that the chosen sets of macrospin parameters are reasonable for all samples. The analysis of the resonance response to an $\mathrm{AC}$ driving magnetic field shows that the full solution of the LLG equation (2) consists of two parts: The first part corresponds to the damped free oscillations with resonance frequencies $\omega_{i}$ [cf. Eq. [11)]. The related terms are transient and disappear after a characteristic time $\propto \frac{1}{\omega_{i}}$. The second part corresponds to the steady-state oscillations with the driving magneticfield frequency $\left(\propto e^{i \omega_{\mathrm{AC}} t}\right)$ 51]. In particular, the amplitude of this term becomes enhanced when its frequency matches the resonance frequencies $\omega_{\mathrm{AC}} \approx \omega_{i}$. Thus, the mode intensities calculated according to Eq. (16) may not be relevant anymore, so that we calculated all resonance modes regardless of their amplitudes. The model predictions were compared with the experimental data.

The VNA-FMR spectra were measured by sweeping the magnetic field at constant frequency of the driving microwave magnetic field $H_{\mathrm{AC}}$. These measurements were repeated for several frequencies of $H_{\mathrm{AC}}$, ranging from 5 to $16 \mathrm{GHz}$ for all samples. The experimental and theoretical results are shown in Figs. 14(a) 14(c).

A detailed analysis reveals that, in addition to clearly visible peaks, abrupt changes also appear in the real and imaginary part of $S_{12}$ for both positive and negative magnetic fields. Such characteristic "jumps" can be assigned to additional resonance peaks. Also, we identify subpeaks within the peaks with larger width. The identification of resonance peaks (resonance fields) is difficult but possible by fitting the relevant Lorentzians, including symmetric

$$
S(H)=\frac{\Delta H^{2}}{\left(H-H_{R}\right)^{2}+\Delta H^{2}}
$$

and antisymmetric

$$
A(H)=\frac{\Delta H\left(H-H_{R}\right)}{\left(H-H_{R}\right)^{2}+\Delta H^{2}}
$$

contributions. Figure 15 shows the $S_{12}$ spectrum of sample $\mathrm{C} 2$ measured at $5 \mathrm{GHz}$. The measured spectrum is one of the most demanding in terms of resonance-mode analysis. Because of the experimental noise, the spectrum is not fully symmetric with respect to $H=0$ (i.e., the amplitude, linewidth, and shape of the resonance peaks differ for positive and negative magnetic fields). For this reason Lorentzians were fit to positive and negative magnetic fields separately. Conversely, the resonance frequencies turned out to be much more symmetric, so they could be analyzed regardless of any differences in other parameters. Such an approach results in a complex relation of dispersions in the case of samples $\mathrm{C} 1$ and $\mathrm{C} 2$, and a relatively simple (Kittel-like) dependency in 


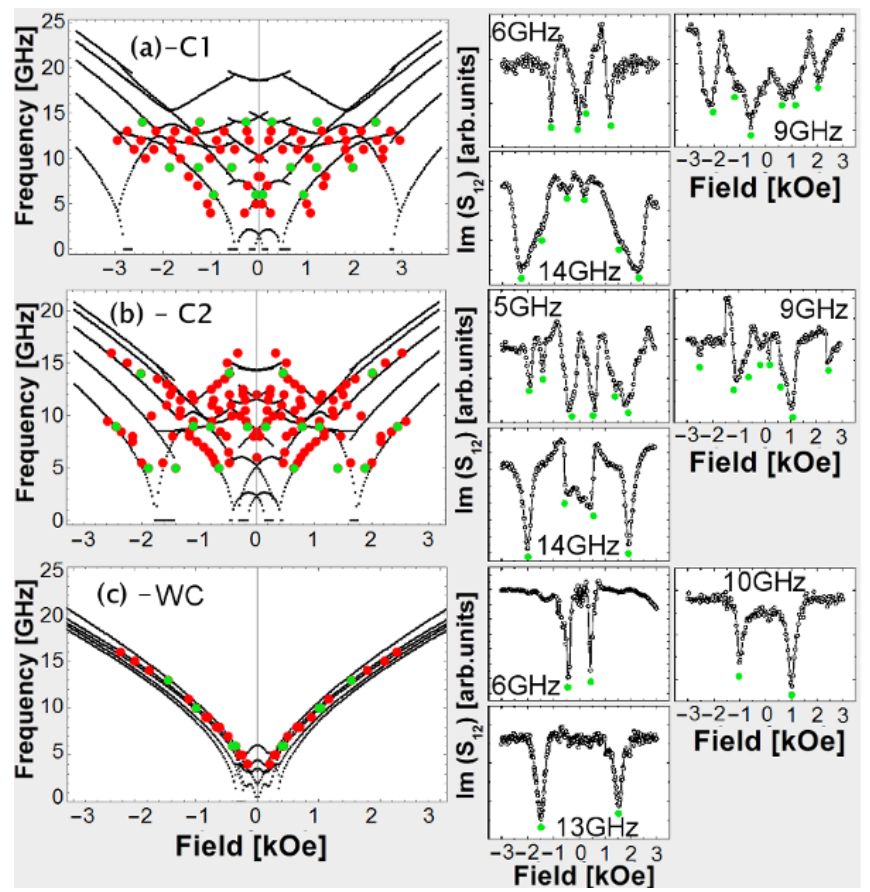

FIG. 14: Left: Experimental resonance fields of samples (a) $\mathrm{C} 1$, (b) $\mathrm{C} 2$, and (c) WC determined from VNA measurements and compared with theoretical (macrospin) dispersion relations. Right: Examples of measured VNA spectra of $S_{12}$ parameter for given frequencies. Green points indicate identified resonance peaks and their counterparts in the dispersionrelation plots. The VNA spectra corresponding to red points are not shown here.

the case of sample WC. For the coupled samples (C1 and $\mathrm{C} 2$ ), the interlayer coupling is responsible for splitting the $f\left(H_{\text {res }}\right)$ branches (resonant modes) and makes the VNA spectra complex. The experimental and theoretical dispersion relations are not reducible to a simple sum of five Kittel-like dispersion relations related to five different Co layers. According to the model, the entire spectrum of resonance fields should be treated as one object, which means that every magnetic (Co) layer interacts through Mo layers with other magnetic (Co) layers, not only with the nearest magnetic neighbors. The influence of the coupling is suppressed when a Mo layer is sufficiently thick. Thus we conclude that the WC sample follows the Kittel formula, so all branches of $f(H)$ converge to the one branch. The splitting (cf. the highest-frequency branch) visible for the WC sample is due to presence of one relatively thick Mo layer and, consequently, a very weak AFM coupling. Moreover, the middle Co layer is considerably thinner than the others. This reason is more important here because the thinness of the middle Co layer causes its $M_{S}$ to be smaller as well. However, the suppressed interlayer coupling makes the VNA resonance peaks easily distinguishable. Note that, for a given frequency, most branch gaps are of the order of the VNA resonance-peak widths. Thus we cannot exclude that more than one narrow peak exists. Such narrow peaks

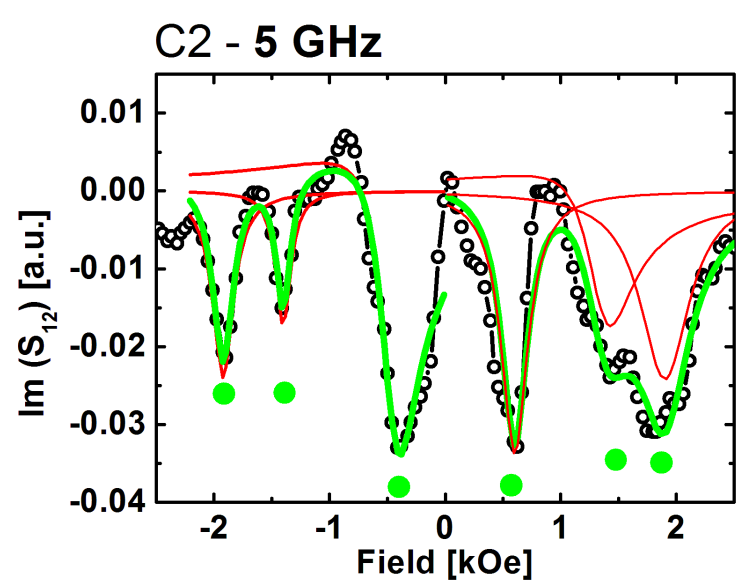

FIG. 15: Experimental VNA spectrum of sample C2 measured under a $5 \mathrm{GHz}$ driving magnetic field. Each separate green line is the sum of three independent Lorentzians corresponding to three distinguishable resonance peaks (red lines). The fitting procedure was done separately for positive and negative magnetic fields.

can be simply fit to the experimental spectra (not shown here), which is obviously justified by a model. However, here we assume a sufficiently wide one-resonance mode covering the five slightly split submodes.

A detailed analysis of oscillation-mode phase (for sample C2) reveals a further abundance of dynamical states. Because of the number of magnetic layers, there are $2^{5}$ different in-phase and antiphase type (symmetries) of oscillation modes. By grouping similar types of oscillations, the number of modes reduces to five with different symmetries denoted by letters $\mathrm{A}$ and $\mathrm{O} 1$ to $\mathrm{O} 4$. Here, the letters $\mathrm{A}$ and $\mathrm{O}$ stand for acoustic and optical modes, respectively; they are illustrated in Fig. 16. Each square corresponds to one Co layer, while its color (gray or red) indicates the phase of the magnetization oscillations, so that the same colors mean the same phase of the magnetization oscillations. As shown in Fig. 16, each mode has a well-established symmetry when the frequency gap between the modes is sufficiently large. In particular, it is visible in low and high magnetic fields, for which a high-frequency mode is acoustic (optical), whereas a lowfrequency mode is optical (acoustic) over a broad range of high (low) magnetic fields. This result is consistent with similar studies of the coupled $[\mathrm{Fe} / \mathrm{Cr}] \times 2 / \mathrm{Fe}$ trilayer structure [52]. However, in the present case, the mode frequencies get closer to each other at intermediate magnetic fields. Moreover, for a certain range of magnetic fields, the modes frequencies overlap; that is, they synchronize their frequencies [cf. two high-frequency modes (red and green points) the moderate modes (green and blue points) or two low-frequency modes (orange and black points)]. In the regions where the modes are close together, multiple changes are observed in their shapes (symmetries) upon increasing the magnetic field. 

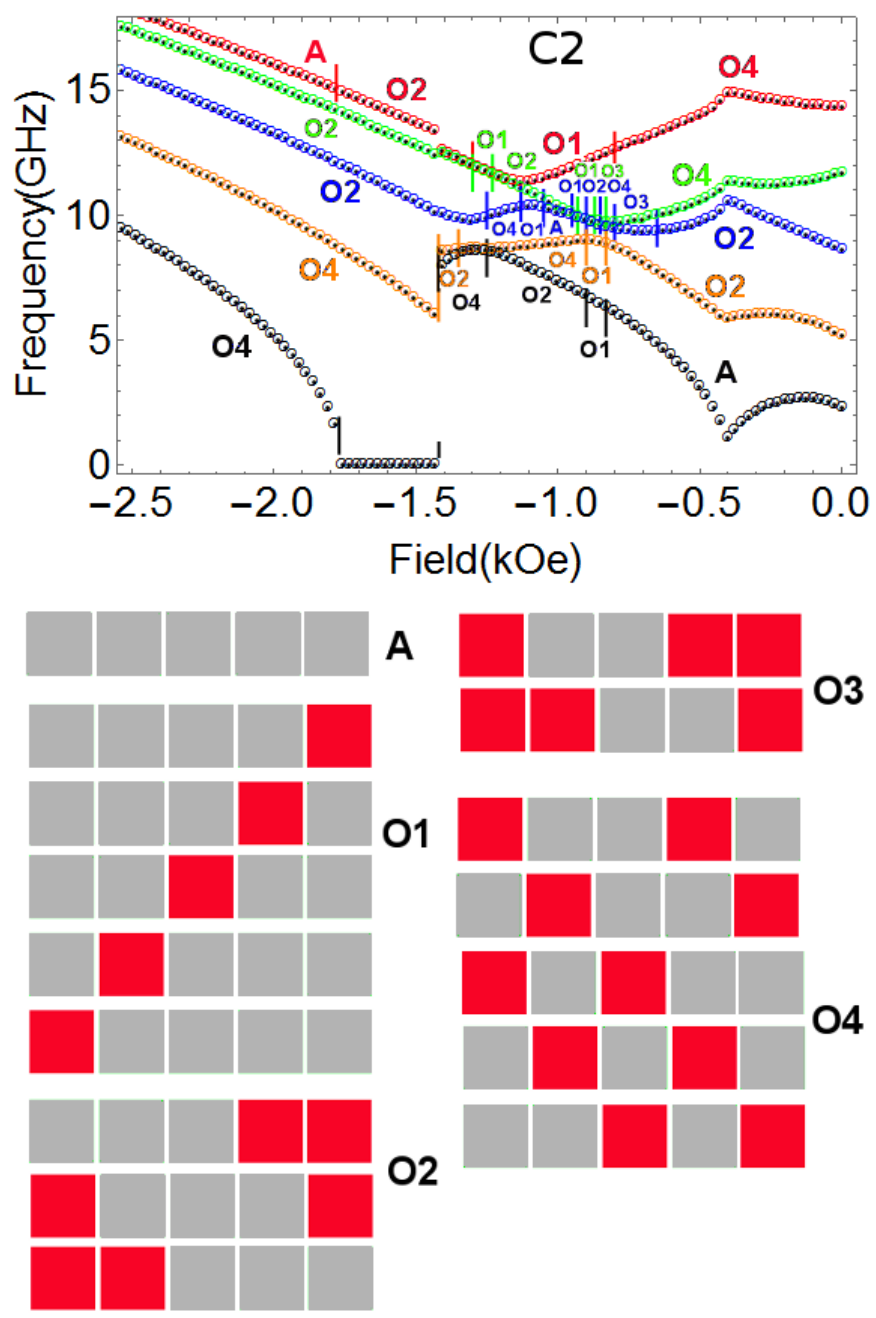

FIG. 16: Top: Five branches of the dispersion relation, each marked by a different color, calculated for sample C2. Different types of optical and acoustic modes marked A and O1 to $\mathrm{O} 4$ with colors assigned to the respective branch. A short vertical colored line indicates the transitions from one type of mode to another within the given branch. Bottom: Illustration of five types of oscillation modes: 1 acoustic and 4 optical modes. Five squares in a row represent the Co sublayers within the sample oscillating with the same frequency, whereas the same colors (gray or red) denote the layers oscillating in the same phase. Each type of modes is realized in one (in the case of A mode) or more $(5,3,2$ in the case of O1 and $\mathrm{O} 4, \mathrm{O} 2, \mathrm{O} 3$ modes, respectively) ways.

\section{Coupling energy}

This section parametrizes the key relations for multilayers (i.e., the coupling energy versus Mo spacer thickness). The relation obtained $\left[J\left(d_{\mathrm{Mo}}\right)\right]$ for our uncompensated SAF is compared with those reported in the literature.

Section $\mathrm{VC}$ explains that interlayer coupling is the main factor affecting the complex magnetic dynamics, and consequently samples $\mathrm{C} 1$ and $\mathrm{C} 2$ reveal qualitatively different dispersion relations than sample WC. Here, we determine how the interlayer coupling magnitude depends on the Mo layer thickness $\left[J\left(d_{\mathrm{Mo}}\right)\right.$. The interlayer coupling magnitude between each subsequent pair of Co sublayers $\left(J_{i j}\right)$ can be determined as one of the fitting parameters (cf. Table III) and from the analysis of the XRR data (cf. Sec. IV). The latter technique reveals the information regarding the Mo layer thickness in all samples. The plot of the semi-empirical points (i.e., $J$ versus $\left.d_{\mathrm{Mo}}\right)$, is shown in Fig. 17. The thickness $d_{\text {Mo }}$ has a maximum relative error of $10 \%$, while the absolute error of $J_{i j}$ is estimated to be $\pm 0.01 \mathrm{~mJ} / \mathrm{m}^{2}$. Such a value for the absolute error ensures that the experimental dependencies of VSM, VNA, and MR are satisfactorily reproduced by the theoretical model.

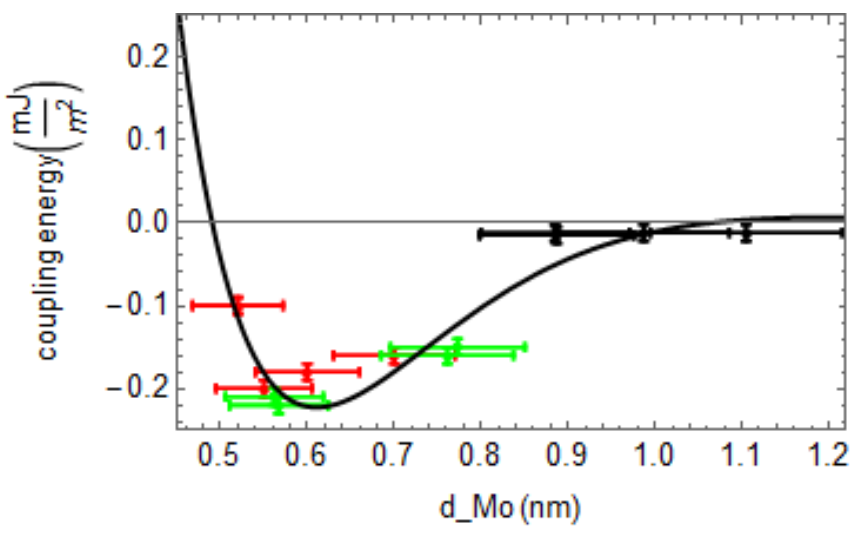

FIG. 17: Coupling energy as a function of Mo layer thickness. The red, green, and black points correspond to Mo thickness determined from XRR measurement for $\mathrm{C} 1, \mathrm{C} 2$ and WC samples respectively. The black solid line is fit to the experimental points.

The theoretical curve was fit to the semi-empirical points by using the RKKY-like interaction [34]:

$$
J\left(d_{\mathrm{Mo}}\right)=\frac{A}{d_{\mathrm{Mo}}^{2}} \sin \left(2 \pi \frac{d_{\mathrm{Mo}}}{\Lambda}+\psi\right) e^{\frac{d_{\mathrm{Mo}}}{t_{c}}},
$$

where $A$ and $\psi$ are the coupling amplitude and phase, respectively, and $\Lambda$ and $t_{c}$ are the period of the coupling oscillations and attenuation length, respectively. The fitting to the semi-empirical points was done with the following parameter values: $A=1.4 \mathrm{~J}, \Lambda=1.15 \mathrm{~nm}$, $\psi=0.45, t_{c}=0.26 \mathrm{~nm}$. The result is shown as a solid line in Fig. 17. A deep minimum around $d_{\mathrm{Mo}} \approx 0.6$ $\mathrm{nm}$ reflects the maximum AFM strength of the interlayer coupling. The obtained parameters are reliable and close to those reported for $\mathrm{Co} / \mathrm{Mo} / \mathrm{Co}$ trilayers 34 and for the system consisting of $16 \mathrm{Co} / \mathrm{Mo}$ bilayers [31]. To compare the results obtained herein with those from Refs. 31, 34, we converted our parameters into the same quantities as used in these references. Their comparison is given in Table IV] Our parameters are located in the middle between the wedge structure and multilayered $\mathrm{Co} / \mathrm{Mo}$ structure. This supports our argument for the reliability of the parametrization of the coupling. 
TABLE IV: Comparison of interlayer coupling parameters for different $\mathrm{Co} /$ Mo structures: five $\mathrm{Co} /$ Mo bilayers (present work), Co/Mo wedge (Ref. 34) and $16 \mathrm{Co} / \mathrm{Mo}$ bilayers (Ref. [31]). $d_{\mathrm{Mo}, \min }^{\mathrm{AFM}}$ : Mo thickness at which the coupling dependence exhibits a first AFM minimum, $J\left(d_{\mathrm{Mo}, \min }^{\mathrm{AFM}}\right)$ : magnitude of coupling at first AFM minimum, $\Lambda$ : period of coupling oscillations, $\Delta d_{\mathrm{Mo}, 1}^{\mathrm{AFM}}$ - range of Mo thickness where the coupling is AFM for the first AFM minimum.

\begin{tabular}{ccccc}
\hline Structure & $\begin{array}{c}d_{\mathrm{Mo}, \mathrm{min}}^{\mathrm{AFM}} \\
{[\mathrm{nm}]}\end{array}$ & $\begin{array}{c}J\left(d_{\mathrm{Mo}, \mathrm{Jin}}^{\mathrm{AFMM}}\right) \\
{\left[\frac{\mathrm{J}}{\mathrm{m}^{2}}\right]}\end{array}$ & $\begin{array}{c}\Lambda \\
{[\mathrm{nm}]}\end{array}$ & $\begin{array}{c}\Delta d_{\mathrm{Mo}, 1}^{\mathrm{AFM}} \\
{[\mathrm{nm}]}\end{array}$ \\
\hline Present work & 0.61 & -0.22 & 1.15 & 0.57 \\
Ref. 34] & 0.7 & -0.33 & 1.40 & 0.71 \\
Ref. 31] & 0.52 & -0.12 & 1.10 & 0.30 \\
\hline \hline
\end{tabular}

\section{SUMMARY AND CONCLUSIONS}

Epitaxially grown Co/Mo multilayers exhibit diverse magnetic properties. The interlayer AFM or FM coupling can be tuned by varying the thickness of the Mo spacer. The superlattices analyzed herein reveal substantial AFM interlayer coupling strength within certain ranges of Mo spacer thickness, making them applicable as uncompensated SAFs (cf. Sec. VD). Moreover, the symmetry and lattice parameter mismatches at the interfaces generate anisotropic strain in the Co layers and, consequently, due to magnetoelastic effects, in-plane twofold magnetic anisotropy is induced. This result allows the mutually orthogonal easy and hard axes of magnetization to be easily determined (cf. Sec. IV). Therefore, in such structures, the magnetization orientation in the sample plane is well defined, which is important for applications. These complex magnetic properties are clearly explained by detailed analyses of the structural properties obtained from complementary techniques (XRD, XRR, and TEM), which are presented in Sec. IV] Moreover, the magnetic configuration of the multilayer sub- stantially affects the magnetization-reversal process (cf. Sec. VB 1. Results of numerical modeling based on macrospin and micromagnetic approaches reproduce well the experimental results and allow us to determine a reliable set of magnetic parameters (cf. Sec. VB1) describing the $\mathrm{Co} / \mathrm{Mo}$ multilayers. In particular, we show that considering the real thickness of component layers is of significant importance for the analysis of magnetodynamic and magnetostatic properties of Co/Mo superlattices. Such a detailed approach allows us to obtain the interlayer-coupling dependence on Mo-spacer thickness (cf. Sec. VD). The coupling is of the RKKY type and is similar to that for Co/Mo sandwiches. Despite a relatively strong interlayer coupling, the MR in multilayers, which is similar to that of a bilayer system, is very weak (cf. Sec. V B 1). The reliable parametrization of the interlayer coupling and of the magnetic-layer properties reveals qualitative differences in dispersion relations in coupled versus WC SAFs (cf. Sec. VC). Finally, the acoustic and optical modes and their evolution with resonance magnetic field were indentified in a strongly coupled SAF (cf. Sec. VC).

\section{ACKNOWLEDGMENTS}

This work was financed by the National Science Centre in Poland under the project: SPINORBITRONICS 2016/23/B/ST3/01430 (acknowledgments from J.K., P.O., S.Z., M.C., and T.S.) and 2014/13/B/ST5/01834 (acknowledgments from A.W., J.K., A.P., K.M., P.D., and K.D.) and co-financed by the EU European Regional Development Fund (REINTEGRATION 2017 OPIE 1420) (acknowledgments from A.W. and A.P.). Numerical calculations were supported in part by the PL-GRID infrastructure. All authors thank S. Łazarski and M. Matczak for their help with VSM and MR measurements.
[1] P. Grünberg, R. Schreiber, Y. Pang, M. B. Brodsky, and H. Sowers, Phys. Rev. Lett. 57, 2442 (1986), URL https: //link.aps.org/doi/10.1103/PhysRevLett.57.2442.

[2] C. F. Majkrzak, J. W. Cable, J. Kwo, M. Hong, D. B. McWhan, Y. Yafet, J. V. Waszczak, and C. Vettier, Phys. Rev. Lett. 56, 2700 (1986), URL https://link.aps. org/doi/10.1103/PhysRevLett.56.2700

[3] M. B. Salamon, S. Sinha, J. J. Rhyne, J. E. Cunningham, R. W. Erwin, J. Borchers, and C. P. Flynn, Phys. Rev. Lett. 56, 259 (1986), URL https://link.aps.org/doi/ 10.1103/PhysRevLett.56.259

[4] C. Carbone and S. F. Alvarado, Phys. Rev. B 36, 2433 (1987), URL https://link.aps.org/doi/10. 1103/PhysRevB.36.2433.

[5] V. Grolier, D. Renard, B. Bartenlian, P. Beauvillain, C. Chappert, C. Dupas, J. Ferré, M. Galtier, E. Kolb, M. Mulloy, et al., Phys. Rev. Lett. 71, 3023 (1993), URL https://link.aps.org/doi/10. 1103/PhysRevLett.71.3023.
[6] P. Bruno and C. Chappert, Phys. Rev. Lett. 67, 1602 (1991), URL https://link.aps.org/doi/10. 1103/PhysRevLett.67.1602

[7] P. Bruno, Phys. Rev. B 52, 411 (1995), URL https: //link.aps.org/doi/10.1103/PhysRevB.52.411

[8] M. N. Baibich, J. M. Broto, A. Fert, F. N. Van Dau, F. Petroff, P. Etienne, G. Creuzet, A. Friederich, and J. Chazelas, Phys. Rev. Lett. 61, 2472 (1988), URL https://link.aps.org/doi/10. 1103/PhysRevLett.61.2472

[9] R. E. Camley and J. Barnaś, Phys. Rev. Lett. 63, 664 (1989), URL https://link.aps.org/doi/10.1103/ PhysRevLett.63.664

[10] J. M. Gomez-Perez, S. Vélez, L. McKenzie-Sell, M. Amado, J. Herrero-Martín, J. López-López, S. Blanco-Canosa, L. E. Hueso, A. Chuvilin, J. W. A. Robinson, et al., Phys. Rev. Applied 10, 044046 (2018), URL https://link.aps.org/doi/10.1103/ PhysRevApplied.10.044046. 
[11] E. Liu, Y.-C. Wu, S. Couet, S. Mertens, S. Rao, W. Kim, K. Garello, D. Crotti, S. Van Elshocht, J. De Boeck, et al., Phys. Rev. Applied 10, 054054 (2018), URL https://link.aps.org/doi/10. 1103/PhysRevApplied.10.054054.

[12] J. Chatterjee, S. Auffret, R. Sousa, P. Coelho, I.-L. Prejbeanu, and B. Dieny, Sci. Rep. 8, 11724 (2018), URL https://doi.org/10.1038/s41598-018-29913-6.

[13] U. Chaudhuri, L. Xiong, R. Mahendiran, and A. O. Adeyeye, Appl. Phys. Lett. 113, 262406 (2018), URL http://doi.org/10.1063/1.5054358.

[14] P. X. Zhang, L. Y. Liao, G. Y. Shi, R. Q. Zhang, H. Q. Wu, Y. Y. Wang, F. Pan, and C. Song, Phys. Rev. B 97, 214403 (2018), URL https://link.aps.org/doi/ 10.1103/PhysRevB.97.214403.

[15] H. Wu, S. A. Razavi, Q. Shao, X. Li, K. L. Wong, Y. Liu, G. Yin, and K. L. Wang, Phys. Rev. B 99, 184403 (2019), URL https://link.aps.org/doi/10. 1103/PhysRevB.99.184403.

[16] M. Farle, Rep. Prog. Phys. 61, 755 (1998), URL https: //doi.org/10.1088\%2F0034-4885\%2F61\%2F7\%2F001.

[17] J. Lindner and K. Baberschke, J. Phys. Condens. Mat. 15, S465 (2003), URL https://doi.org/10.1088\% 2F0953-8984\%2F15\%2F5\%2F303.

[18] B. Khodadadi, J. B. Mohammadi, J. M. Jones, A. Srivastava, C. Mewes, T. Mewes, and C. Kaiser, Phys. Rev. Applied 8, 014024 (2017), URL https://link.aps .org/ doi/10.1103/PhysRevApplied.8.014024.

[19] C. R. Rementer, K. Fitzell, Q. Xu, P. Nordeen, G. P. Carman, Y. E. Wang, and J. P. Chang, Appl. Phys. Lett. 110, 242403 (2017), URL https://doi .org/10.1063/1. 4984298

[20] C. G. Bezerra, J. M. de Araújo, C. Chesman, and E. L. Albuquerque, Phys. Rev. B 60, 9264 (1999), URL https : //link.aps.org/doi/10.1103/PhysRevB.60.9264

[21] M. Sousa, J. Quispe-Marcatoma, F. Pelegrini, and E. Baggio-Saitovitch, J. Magn. Magn. Mater. 474, 250 (2019), URL http://doi.org/10.1016/j.jmmm.2018. 10.127 .

[22] S. Li, G.-X. Miao, D. Cao, Q. Li, J. Xu, Z. Wen, Y. Dai, S. Yan, and Y. Lü, ACS Appl. Mater. Interfaces 10, 8853 (2018), URL https://doi.org/10.1021/acsami. $7 \mathrm{~b} 19684$

[23] A. Hrabec, N. A. Porter, A. Wells, M. J. Benitez, G. Burnell, S. McVitie, D. McGrouther, T. A. Moore, and C. H. Marrows, Phys. Rev. B 90, 020402 (2014), URL https: //link.aps.org/doi/10.1103/PhysRevB.90.020402

[24] A. Fert, N. Reyren, and V. Cros, Nature Reviews Materials 2, 17031 (2017), URL https://doi.org/10.1038/ natrevmats.2016.44.

[25] B. Zhao, G. Yang, F. Zeng, and F. Pan, Acta Mater. 51, 5093 (2003), URL https://doi.org/10. 1016/S1359-6454(03)00359-8

[26] G. Yang, Y. Gu, Y. Gao, and F. Pan, Thin Solid Films 457, 354 (2004), URL https://doi.org/10.1016/ j.tsf.2003.11.290.

[27] G. Yang and F. Pan, J. Magn. Magn. Mater. 250, 249 (2002), URL https://doi.org/10.1016/ S0304-8853(02)00402-X

[28] Y. Wang, F. Cui, W. Li, and Y. Fan, J. Magn. Magn. Mater. 102, 121 (1991), URL https://doi.org/10. 1016/0304-8853(91)90276-G.

[29] J. Houserová, J. Vřešt'ál, and M. Šob, Calphad 29, 133 (2005), URL https://doi.org/10.1016/j. calphad.2005.06.002

[30] H. Guo, L. Kong, J. Liu, and B. Liu, Solid State Commun. 125, 435 (2003), URL https://doi.org/10.1016/ S0038-1098(02) 00824-4

[31] S. S. P. Parkin, Phys. Rev. Lett. 67, 3598 (1991), URL https://link.aps.org/doi/10.1103/PhysRevLett.67. 3598

[32] E. Shalyguina, E. Perepelova, L. Kozlovskii, and E. Tamanis, J. Magn. Magn. Mater. 300, e363 (2006), URL http://www.sciencedirect.com/science/ article/pii/S0304885305009704.

[33] D. D. Koelling, Phys. Rev. B 50, 273 (1994), URL https : //link.aps.org/doi/10.1103/PhysRevB.50.273

[34] Z. Kurant, M. Tekielak, I. Sveklo, A. Wawro, and A. Maziewski, J. Magn. Magn. Mater. 475, 683 (2019), ISSN 0304-8853, URL http://www.sciencedirect.com/ science/article/pii/S0304885318335984

[35] A. Wawro, Z. Kurant, M. Tekielak, P. Nawrocki, E. Milińska, A. Pietruczik, M. Wójcik, P. Mazalski, J. Kanak, K. Ollefs, et al., J. Phys. D Appl. Phys. 50, 215004 (2017), URL https://doi.org/10.1088/ 1361-6463/aa6a94

[36] A. Wawro, Z. Kurant, M. Tekielak, M. Jakubowski, A. Pietruczik, R. Böttger, and A. Maziewski, Appl. Phys. Lett. 110, 252405 (2017), URL https://doi.org/10. $1063 / 1.4987142$.

[37] A. Wawro, Z. Kurant, M. Jakubowski, M. Tekielak, A. Pietruczik, R. Böttger, and A. Maziewski, Phys. Rev. Applied 9, 014029 (2018), URL https://link.aps .org/ doi/10.1103/PhysRevApplied.9.014029.

[38] A. Wawro, E. Milińska, Z. Kurant, A. Pietruczik, J. Kanak, K. Ollefs, F. Wilhelm, A. Rogalev, and A. Maziewski, J. Synchrotron Rad. 25, 1400 (2018), URL https://doi.org/10.1107/S1600577518008500

[39] I. Sveklo, Z. Kurant, M. Tekielak, A. Pietruczik, K. Dybko, A. Wawro, and M. A., J. Magn. Magn. Mater. 489, 165417 (2019), URL http://www.sciencedirect.com/ science/article/pii/S0304885319304238

[40] M. J. Donahue and D. G. Porter, OOMMF User's guide (US Department of Commerce, Technology Administration, National Institute of Standards and Technology, 1999).

[41] J. Kanak, P. Wiśniowski, T. Stobiecki, A. Zaleski, W. Powroźnik, S. Cardoso, and P. P. Freitas, J. Appl. Phys. 113, 023915 (2013), https://doi.org/10.1063/1.4775706, URL https: //doi.org/10.1063/1.4775706

[42] J. Prokop, D. A. Valdaitsev, A. Kukunin, M. Pratzer, G. Schönhense, and H. J. Elmers, Phys. Rev. B 70, 184423 (2004), URL https://link.aps.org/doi/10. 1103/PhysRevB.70.184423

[43] J. Smit and H. Beljers, Philips Res. Rep. 10, 1113 (1955).

[44] E. Barati, M. Cinal, D. M. Edwards, and A. Umerski, Phys. Rev. B 90, 014420 (2014), URL https://link. aps.org/doi/10.1103/PhysRevB.90.014420.

[45] P. Ogrodnik, F. A. Vetrò, M. Frankowski, J. Chęciński, T. Stobiecki, J. Barnaś, and J.-P. Ansermet, J. Phys. D Appl. Phys. 52, 065002 (2018), URL https://doi.org/ $10.1088 \% 2 \mathrm{~F} 1361-6463 \% 2 \mathrm{Faaf} 093$

[46] M. Frankowski, J. Chęciński, and M. Czapkiewicz, Computer Physics Communications 189, 207 (2015), ISSN 0010-4655, URL http://www.sciencedirect.com/ science/article/pii/S0010465514004159

[47] P. Bloemen, Acta Phys. Pol. A 89, 277 (1996). 
[48] A. Shukh, D. Shin, and H. Hoffmann, J. Appl. Phys. 76, 6507 (1994), URL https://doi.org/10.1063/1.358244

[49] T. McGuire and R. Potter, IEEE Trans. Mag. 11, 1018 (1975), URL https://doi.org/10.1109/TMAG. 1975.1058782

[50] A. Drovosekov, D. Kholin, N. Kreines, O. Zhotikova, and S. Demokritov, Journal of Magnetism and Magnetic Materials 226-230, 1779 (2001), ISSN 0304-8853, proceedings of the International Conference on Magnetism (ICM
2000), URL http://www.sciencedirect.com/science/ article/pii/S0304885300010131.

[51] H. J. Pain and R. T. Beyer, The physics of vibrations and waves (ASA, 1993).

[52] R. Zivieri, L. Giovannini, and F. Nizzoli, Phys. Rev. B 62, 14950 (2000), URL https://link.aps.org/doi/10. 1103/PhysRevB.62.14950. 